\title{
A BEM-ISOGEOMETRIC method for the ship wave-resistance problem
}

\author{
K.A. Belibassakis ${ }^{(1, *)}$, Th.P. Gerostathis ${ }^{(2)}$, K.V. $\operatorname{Kostas}^{(2)}$, C.G. Politis ${ }^{(2)}$ \\ P.D. Kaklis ${ }^{(1)}$, A.I. Ginnis ${ }^{(1)}$ and C. Feurer ${ }^{(1)}$ \\ (1) School of Naval Architecture and Marine Engineering, \\ National Technical University of Athens, Zografos 15773, Athens, Greece. \\ ${ }^{(2)}$ Dept. of Naval Architecture, Technological Educational Institute of Athens \\ Ag. Spyridonos 12210, Athens, Greece
}

\begin{abstract}
In the present work IsoGeometric Analysis is applied to the solution of the Boundary Integral Equation associated with the Neumann-Kelvin problem and the calculation of the wave resistance of ships. As opposed to low-order panel methods, where the body is represented by a large number of quadrilateral panels and the velocity potential is assumed to be piecewise constant (or approximated by low degree polynomials) on each panel, the isogeometric concept is based on exploiting the same NURBS basis, used for representing exactly the body geometry, for approximating the singularity distribution (and, in general, the dependent physical quantities). In order to examine the accuracy of the present method, numerical results obtained in the case of submerged and surface piercing bodies are
\end{abstract}

\footnotetext{
Corresponding author. Tel: (+30) 2107721138, Fax: (+30) 2107721397, e-mail: kbel@fluid.mech.ntua.gr
} 
compared against analytical solutions, experimental data and predictions provided by the low-order panel or other similar methods appeared in the pertinent literature, illustrating the superior efficiency of the isogeometric approach. The present approach by applying Isogeometric Analysis and Boundary Element Method to the linear NK problem has the novelty of combining modern CAD systems for ship-hull design with computational hydrodynamics tools.

Keywords: Isogeometric Analysis, high-order BEM, Neumann-Kelvin problem, NURBS, CAD-CFD integration

\section{INTRODUCTION}

Due to its specific importance in ship powering prediction and optimisation of ship hulls, the investigation of ship resistance in calm water is a significant problem. This problem is complicated since ship resistance is dependent on both viscous and gravitational effects. For computational purposes, the calculation of viscous and wave-making resistance is usually considered separately. Wave-making resistance is a very important component, which sometimes can contribute $50 \%$ or even more of the total resistance of a ship (especially for relatively full hull forms and/or at high speeds). Experience has shown that the wave-making resistance component is quite sensitive to design parameters and significant reduction can be achieved without affecting cargo capacity. The capability to predict and minimize wave resistance in the early stages of the design is therefore very important.

During the last 50 years, the interest in numerical methods for calculating ship wave resistance has been constantly growing. Computations are performed using a variety of techniques, ranging from the simple Michell's thin ship theory to fully non-linear Reynolds-Averaged-Navier-Stokes Equations (RANSE) methods; recent advances are 
presented in the reports by ITTC; see ITTC $(2005,2008)$ and the references cited therein. The application of three-dimensional potential flow theory to the steady ship motion problem results in an essentially non-linear boundary value problem (due to the non-linear character of the boundary condition at the free surface), from which the unknown velocity potential and the free-surface disturbance must be calculated. The Boundary Element Method (BEM) is a widely used approach to solve potential flow problems in marine hydrodynamics, with further application to the calculation of the wave resistance and the wave pattern of ships steadily advancing at forward speed. There exist two main types of elementary singularities used in the implementation of the method. The first type uses the Kelvin wave source as the elementary singularity, satisfying the field equation and all the boundary conditions except the body-boundary condition. The major advantages of such a scheme are the automatic satisfaction of the radiation condition and the definition of the resulting BIE only on the ship hull; see e.g., Wehausen (1973). The second type uses the simple Rankine source, i.e., the fundamental solution of the Laplace equation, as the elementary singularity. The method was first presented by Dawson (1977) and since then it has been widely applied as a practical method to predict wave resistance. Many improvements have also been made to account for non-linear effects; see e.g., Nakos \& Sclavounos (1990), Raven (1996), Bertram (2000), Bal (2008). Considerable efforts have been devoted to increase efficiency and accuracy by introducing several variations, such as the desingularized method and the RAPID method. The above methods have the advantage of employing a simple elementary singularity, but on the other hand, the resulting Boundary Integral Equation (BIE) is extended over the ship hull and part of the unlimited free surface leading to increased computational requirements.

In the present work, IsoGeometric Analysis (IGA), proposed by Hughes et al $(2005,2008)$ and used in the context of Finite Element Method (see, e.g., Cottrell et al 2007), is applied to the solution of the BIE associated with the linearized Neumann-Kelvin (NK) problem, 
with application to the wave-making resistance of ships and submerged bodies at constant forward speed. A similar boundary-integral approach, concerning problems governed by the Laplace equation in infinite domains, has been presented in Politis et al (2009) and for linear elastostatics and shape optimization problems in Li \& Qian (2011). In the NK problem, the non-linear effects stemming from the presence of the unknown free surface are neglected, while the three-dimensional character of the fluid flow is fully retained. Independent of its own practical interest, the robust and accurate solution of this linear problem represents a useful first stage before dealing with the complete non-linear problem. For the solution we shall use a BEM implemented by means of a Kelvin wave source distribution over the wetted part of the hull. Fulfilment of the body-boundary condition leads to an integral equation with support only on the wetted part of the hull and its intersection with the unperturbed free surface; see, e.g., Brard (1972) and Baar \& Price (1988).

Integral equation formulation of Laplace boundary-value problems has been established as one of the standard tools for calculating inviscid, incompressible flow characteristics (velocity and pressure) around 2D and 3D bodies and geometrical systems; see, e.g., Hess (1975), Katz-Plotkin (1991). Some of the most important advantages of this approach include the reduction of dimensionality, facilitation of calculations around complex geometrical configurations (especially in 3D), consistent handling of conditions at infinity, high convergence rates when the domain boundary and boundary data are (relatively) smooth and easy implementation to optimization (inverse-type) problems. As concerns the numerical solution, BEM (or panel methods) serve today as valuable tools, especially for non-linear and time-dependent problems; see, e.g., Brebbia et al (1984), Paris \& Canas (1997), Brebbia (2002).

In low-order BEM the body surface is usually discretized by a finite number of elements or patches, each carrying a simple distribution of the unknown function; see, e.g., Hess 
(1975). On the contrary, high-order BEM, characterised by an increased order of approximation both with respect to geometry and the surface singularity distribution, has the property of faster convergence as element-size diminishes, and yields more accurate results with coarser grid resolutions; see, e.g., Gennaretti et al (1998). The latter is found to be quite important especially at places/subregions where the solution presents physical discontinuities and/or singularities, which are not well treated by low-order methods. In this direction, high-order panel methods based on B-spline and/or non-uniform rational Bspline (NURBS) representations have recently appeared in the literature, for potential-flow problems. In the sequel, we briefly present some of these works. The flow around 2D bodies moving with constant speed under the free surface is studied by Okan \& Umpleby (1985) using B-splines. A 3D method for wave-body interaction through a Rankine boundary element approach, based on Maniar (1995) and satisfying the body-boundary condition directly on the exact CAD surfaces, has been applied to multi-body seakeeping design optimization by Peltzer et al (2008). The high-order BEM overlays the NURBS geometry and yields stable wave and motion integration in time. Kring (1995) and Kim \& Shin (2003) solve the three-dimensional radiation and diffraction problem using a NURBS representation of the body geometry and a B-spline basis for the unknown potential. Datta \& Sen $(2006,2007)$ solve, in the time domain, the three-dimensional ship motions problem with forward speed. The problem is formulated using the transient free-surface Green function. The body geometry is represented by either B-spline or NURBS, depending on the hull type, whereas the unknown field variables are represented via B-spline basis functions. Moreover, Kim et al (2007) use a higher-order panel method, based on B-spline representation for both the geometry and the solution, with application to the analysis of steady flow around marine propellers. Also, Gao \& Zou (2008) solve the 3-D radiation and diffraction problem with forward speed, using the Rankine source distribution method, in conjunction with a NURBS surface to precisely represent the body geometry, whereas the velocity potential on the body surface is represented by B-splines. 
As opposed to high-order BEM methods outlined above, where the body and the associated physical quantities are expressed via different basis functions, the IGA approach (see, e.g., Hughes et al 2005, 2008, Cottrell et al 2007, 2009) is based on using the very same basis for representing both the body geometry and the physical quantities, under the constraint that the chosen basis is able to represent accurately the geometry in question. In a recent work by Politis et al (2009), the IGA-BEM is exploited for the solution of the exterior Neumann problem in 2D, governed by the Laplace equation, reformulated as a boundary integral equation by using source-sink formulation. Numerical results obtained for a circular and a free-form contour, represented exactly as NURBS curves, and various types of forcing, are compared against analytical and benchmark solutions. The error is compared against low- and higher-order panel method predictions, illustrating very high rates of algebraic convergence, ranging from $O\left(N^{-4}\right)$ to $O\left(N^{-10}\right)$, if mesh refinement, through knot insertion, is combined with degree elevation, where $N$ is the number of degrees of freedom. This result, compared with the rate $O\left(N^{-1}\right)$ of the low-order panel method, is found to be promising for BIE of more complicated flow problems, like the ones associated with ship and submerged bodies in steady motion. Thus, in this paper, the IGA-BEM is applied to the wave-making resistance problem of surface piercing bodies and ships. The present approach, although focusing on the linear NK problem, has the novelty of combining modern CAD systems for ship-hull design with computational hydrodynamics tools.

The rest of the paper is structured in four sections. Sec. 2 summarizes the formulation of the NK problem as a BIE. In Sec. 3 we develop in four subsections an isogeometricallyoriented BEM for solving the BIE formulated in Sec. 2. More specifically, in $\S 3.1$ we introduce the multi-patch NURBS representation and illustrate it for a triplet of geometries that are used later in Sec. 4 for testing the proposed method. In $\S 3.2$ we introduce the 
isogeometric treatment of the present BIE, while $\S 3.3$ discusses the issue of stable numerical handling of the involved integrals. Finally, in $\S 3.4$ we formulate the linear system arising from discretizing the BIE of Sec. 2 in a collocation setting and discuss alternative refinement approaches for converging towards the continuous solution. Subsequently, Sec. 4 is devoted to presenting and discussing the performance of the proposed IGA-BEM for solving the NK problem for a variety of geometries and physical configurations. The material starts with two simple tests, namely a prolate spheroid moving with constant speed in infinite homogeneous fluid (§ 4.1) and a submerged ellipsoid moving at high speed under the free surface $(\S 4.2)$. Subsection 4.3 presents results from testing the proposed method in the case of a submerged prolate spheroid moving steadily under the free surface, while the next two subsections deal with surface-piercing hulls, namely a Wigley parabolic hull $(\S 4.4)$ and a Series 60 hull $(\S 4.5)$. The obtained results are compared versus a variety of results available in the pertinent literature, e.g., analytical solutions, experimental data and predictions provided by the low-order panel or other similar techniques, illustrating the enhanced accuracy and efficiency of the present method.

\section{FORMULATION OF THE PROBLEM}

We consider a surface-piercing body moving with constant forward speed $U$ in an ideal (homogeneous, incompressible, inviscid) fluid of infinite depth, bounded above by a free surface. In the body-fixed coordinate system $O x y z$, with $z$ - axis pointing vertically upwards (see Fig. 1) this problem is equivalent to a uniform stream with velocity $\mathbf{U}=(-U, 0,0)$ incident on the body $D$. Following the formulation by Brard (1972) and Baar \& Price (1988) for the linearized NK problem, the total flow field is decomposed to the parallel inflow and the disturbance potential which satisfies the Laplace equation,

$\Delta \varphi=0, \quad$ in $\quad D^{+}$, 
where $D^{+}$is the fluid domain outside $D$, limited above by the free surface. Eq. (1) is supplemented by the body-boundary condition,

$\partial \varphi / \partial n=-\mathbf{U} \cdot \mathbf{n}, \quad$ on $\quad S$,

where $\mathbf{n}=\left(n_{x}, n_{y}, n_{z}\right)$ denotes the unit normal vector on $S$ directed outwards the body and $S=\partial D$ denotes the wetted boundary of $D$, the kinematic condition on the free surface,

$\left(-U+\varphi_{x}\right) \eta_{x}+\varphi_{y} \eta_{y}-\varphi_{z}=0, \quad$ on $\quad z=\eta(x, y)$

the dynamic free-surface condition tating that the pressure on the free surface must be constant

$g \eta-U \varphi_{x}+(1 / 2)\left(\varphi_{x}^{2}+\varphi_{y}^{2}+\varphi_{z}^{2}\right)=0, \quad$ on $\quad z=\eta(x, y)$,

and appropriate conditions at infinity. In the formulation of the NK problem, the above conditions on the free surface are linearized, by neglecting higher-order quantities and by applying the resulting equations on the undisturbed free surface $z=0$. Eqs.(2b,c) after linearization, are combined to the following linearized free-surface boundary condition:

$\varphi_{x x}+\left(g / U^{2}\right) \varphi_{z}=0, \quad$ on $\quad z=0$

Thus, the linearized NK problem consists of Eqs.(1),(2),(3), in conjunction with the radiation condition, expressing the fact that the disturbance potential decays at infinity and there are no upstream waves. In the framework of potential theory (see also Baar \& Price 1988, Marr \& Jackson 1999), the disturbance field $\varphi$ is represented by

$\varphi(P)=\int_{S} \mu(Q) G(P, Q) d S(Q)+k^{-1} \int_{\ell} \mu(Q) G^{*}(P, Q) n_{x}(Q) \tau_{y}(Q) d \ell(Q)$,

where $\ell$ denotes the waterline (corresponding to the intersection of $S$ with the undisturbed free-surface $z=0), \quad \mu$ is the density of the source-sink distribution on $S$ and, finally, $g$ denotes the acceleration due to gravity. In the above equation $G$ denotes the NK- Green's function which is defined as

$$
4 \pi G(P, Q)=r^{-1}-R^{-1}+G^{*}(P, Q), \quad Q \in S, \quad P \in \mathbb{R}_{-}^{3} \backslash D,
$$


where $\mathbb{R}_{-}^{3}=\{x, y, z \leq 0\}$ is used to denote the half space below the undisturbed free surface $(z=0), \quad r=|Q P|, \quad R=\left|Q^{\prime} P\right|$, where $Q^{\prime}$ is the image of $Q$ with respect to the $z=0$ plane and $G^{*}(P, Q)$ stands for the regular part of the NK Green's function, consisting of components exhibiting z-exponential decaying and wavelike behavior (see Baar \& Price 1988). Moreover, $\boldsymbol{\tau}=\left(\tau_{x}, \tau_{y}, \tau_{z}\right)$ the corresponding tangent vector along the waterline $\ell$, directed as shown in Fig.1.

The integral representation of the disturbance potential $\varphi$, provided by Eqs. (4), permits us to automatically satisfy the Laplace equation, in conjunction with the linearized condition on the undisturbed free surface and the conditions at infinity. Substitution of integral representation, Eq. (4a), to the body boundary condition, Eq.(2a), and taking into account the jump property of the normal derivative of the single-layer potential, leads to the equivalent reformulation of the NK problem as a BIE defined on the wetted surface $S$, as follows

$$
\begin{array}{r}
\frac{\mu(P)}{2}-\int_{S} \mu(Q) \frac{\partial G(P, Q)}{\partial n(P)} d S(Q)-\frac{1}{k} \int_{\ell} \mu(Q) \frac{\partial G^{*}(P, Q)}{\partial n(P)} n_{x}(Q) \tau_{y}(Q) d \ell(Q)=-\mathbf{U} \cdot \mathbf{n}(P), \\
P, Q \in S .
\end{array}
$$

The quantity $k=g / U^{2}$ is the characteristic wavenumber, controlling the wavelength of the transverse ship waves, that is directly connected with the squared inverse of the corresponding Froude number $F=U / \sqrt{g L}$, with $L$ denoting the max length of the body. From the solution of the above integral equation, various quantities, such as velocity, pressure distribution and ship wave pattern can be obtained. Specifically, total flow velocity and pressure are calculated through the formulae 
$\mathbf{v}=\mathbf{U}+\nabla \varphi, \quad p=p_{\infty}+\frac{\rho}{2}\left(U^{2}-v^{2}\right)-\rho g z$

where $\rho$ denotes the fluid density. Moreover, the free-surface elevation is obtained as

$\eta(x, y)=(U / g) \cdot \varphi_{x}(x, y ; z=0)$.

In the case of a fully submerged body the above formulation should be modified by dropping the waterline integral in Eqs. (4a) and (5).

\section{THE ISOGEOMETRIC BEM}

The IGA philosophy is based on approximating the field quantities (dependent variables) of the boundary-value problem in question by the very same basis that is being used for representing the geometry of the involved body-boundary. In the case of BIE (5), the dependent variable is the source-sink density $\mu$, distributed over the surface $S$. The latter is accurately represented as a parametric NURBS surface or a collection of smoothly joined NURBS patches - referred to as a multi-patch NURBS surface. In this connection it should be stressed that, although NURBS is not a prerequisite IGA ingredient, we adopt it because it is a thoroughly developed and widespread tool in CAD technology.

\subsection{Representation of body geometry}

To proceed we assume that $S$ is a multi-patch NURBS surface $\mathbf{x}\left(t_{1}, t_{2}\right)$, represented as

$$
\mathbf{x}\left(t_{1}, t_{2}\right)=\sum_{i_{1}=0}^{n_{1}^{p}} \sum_{i_{2}=0}^{n_{2}^{p}} \mathbf{d}_{i_{1} i_{2}}^{p} R_{i_{1}, k_{2}, k_{1}^{p} k_{2}^{p}}^{p}\left(t_{1}, t_{2}\right):=\sum_{\mathbf{i}=0}^{n^{p}} \mathbf{d}_{\mathbf{i}}^{p} R_{\mathbf{i}}^{p}\left(t_{1}, t_{2}\right),
$$

and 


$$
\begin{array}{r}
R_{i_{1}, i_{2}, k_{1}^{p} k_{2}^{p}}^{p}\left(t_{1}, t_{2}\right):=R_{\mathbf{i}}^{p}\left(t_{1}, t_{2}\right)=\frac{w_{i_{i} i_{2}}^{p} N_{i_{1}}^{p}\left(t_{1}\right) N_{i_{2}}^{p}\left(t_{2}\right)}{\sum_{l_{1}=0}^{n_{1}^{p}} \sum_{l_{2}=0}^{n_{2}^{p}} w_{l_{1} l_{2}}^{p} N_{l_{1}}^{p}\left(t_{1}\right) N_{l_{2}}^{p}\left(t_{2}\right)}, \quad\left(t_{1}, t_{2}\right) \in I_{1}^{p} \times I_{2}^{p}, \\
p=1,2, \cdots, N,
\end{array}
$$

where $\mathbf{i}=\left(i_{1}, i_{2}\right)$ and $\mathbf{k}^{p}=\left(k_{1}^{p}, k_{2}^{p}\right)$ are multiple indices, $p$ is the patch identifier, $\mathbf{d}_{\mathbf{i}}^{p}:=\mathbf{d}_{i_{1} i_{2}}^{p} \quad$ denote the control points of patch $p$, and $N_{i_{1}}^{p}\left(t_{1}\right):=N_{i_{1}, k_{1}^{p}}^{p}\left(t_{1}\right)$, $N_{i_{2}}^{p}\left(t_{2}\right):=N_{i_{2}, k_{2}^{p}}^{p}\left(t_{2}\right)$ are the B-spline basis functions of degree $k_{1}^{p}$ and $k_{2}^{p}$, respectively. The latter, in conjunction with the weights $w_{i_{1} i_{2}}^{p}$, are used in (7b) for building up the rational B-spline functions $R_{\mathbf{i}}^{p}\left(t_{1}, t_{2}\right)$ for patch $p$. Bold index notation is used to avoid appearance of multiple indices, while the subscript $\mathbf{k}^{p}$ will be henceforth omitted for the sake of simplicity and without loss of accuracy. Furthermore, the parametric intervals $I_{1}^{p}$, $I_{2}^{p}$ are partitioned appropriately by knot vectors $J_{1}^{p}$ and $J_{2}^{p}$, respectively. Finally, in the case of surface piercing bodies, we shall assume that the union of the isoparametric segments $\mathbf{x}\left(t_{1}=t_{1}^{E n d}, t_{2}\right)$ of the uppermost patches $(\hat{p})$ provides the waterline $(\ell)$.

As a first example, the NURBS representation for the ellipsoid can be generated by applying a shear transformation to a bi-quadratic NURBS representation of the sphere given in Piegl \& Tiller (1997). Multi-patch models of the ellipsoid in Fig. 2(a), composed from 2 and 4 patches (see Figs. 2(b) and 2(c)), have been used for testing the multi-patch version of the code. The second example involves the B-spline representation of the classical parabolic Wigley hull, defined analytically as

$$
\frac{y}{B / 2}=\left(1-\left(\frac{x}{L / 2}\right)^{2}\right)\left(1-\left(\frac{z}{T}\right)^{2}\right),
$$


where $L, B, T$ denote the length, breadth and draft of the hull. The single and multi-patch representations of this hull are depicted in Fig. 3. The single patch is generated by interpolation of the bow/stern profiles and a parabola representing the hull mid section. Multi-patch NURBS representations of more complex ship hull geometries and geometrical configurations can be obtained via CAD tools and techniques. For example, the B-spline representation of a Series $60(\mathrm{Cb}=0.60)$ hull, which is discussed below in $\S 4.4$, is obtained using multiple patches, as illustrated in Fig. 4. The semi-hull is a $G^{1}$ continuous surface, i.e., a continuous surface with continuously varying unit normal, comprising 7 bicubic patches with a total count of 3285 control points. In this case, patches 1 and 2 have been generated with a lofting (skinning scheme) on the corresponding ship sections while the remaining patches (3 to 7) are the result of Gordon surface construction schemes on corresponding sections and waterline and/or stern-profile parts.

\subsection{The IGA - boundary integral equation method}

In IGA context the unknown source-sink surface distribution $(\mu)$ is approximated via the very same NURBS basis used for the body boundary representation (Eqs. 7), that is:

$\mu\left(t_{1}, t_{2}\right)=\sum_{\mathbf{i}=0}^{\mathbf{n}^{p}+1^{p}} \mu_{\mathbf{i}}^{p} R_{\mathbf{i}, 1^{p}}^{p}\left(t_{1}, t_{2}\right), \quad\left(t_{1}, t_{2}\right) \in I_{1}^{p} \times I_{2}^{p}, \quad p=1,2, \ldots N$

where $\mu_{\mathrm{i}}^{p}$ are the (unknown) coefficients associated with the above expansion, and $\mathbf{I}^{p}=\left(l_{1}^{p}, l_{2}^{p}\right)$ denotes the additional knots with which the initial knot vectors $J_{1}^{p}$ and $J_{2}^{p}$ are enhanced in order to refine, by knot insertion, the initial NURBS basis and increase 
its approximation power. The totality $M=d o f=\sum_{p}\left(n_{1}^{p}+l_{1}^{p}+1\right)\left(n_{2}^{p}+l_{2}^{p}+1\right)$ of coefficients $\mu_{\mathrm{i}}^{p}$ constitutes the degrees of freedom (dof) associated with approximation (8).

Thus, sequences of spline spaces $\mathbb{S}^{p}\left(J_{1, l_{1}^{p}}^{p}, J_{2, l_{2}^{p}}^{p}\right):=\mathbb{S}_{1}^{p}$ can be produced, on which the boundary integral equation (5) will be projected. These spaces are nested, i.e., $\mathbb{S}_{1}^{p} \subset \mathbb{S}_{\mathbf{k}}^{p}$ if $l_{j}^{p}<k_{j}^{p}, j=1,2$. Several methods are available for defining a projection onto the finitedimensional space $\mathbb{S}_{1}^{p}$ and discretizing Eq. (5), like Galerkin and collocation methods (see, e.g., Kress 1989, Sec.13). In the present work, a collocation scheme is adopted for projecting (5) on $\mathbb{S}_{\mathbf{1}}^{p}$. For this, let $P_{\mathbf{j}}^{p}=P_{j_{1} j_{2}}^{p}=\mathbf{x}\left(t_{1, j_{1}}^{p}, t_{2, j_{2}}^{p}\right)$ denote a set of collocation points on $S$, where $\left\{t_{1, j_{1}}^{p}\right\}, j_{1}=0, \ldots, n_{1}^{p}+l_{1}^{p}$, and $\left\{t_{2, j_{2}}^{p}\right\}, j_{2}=0, \ldots, n_{2}^{p}+l_{2}^{p}$, lie in $I_{1}^{p}$ and $I_{2}^{p}$, respectively, for $p=1,2, \ldots N$. For each patch $p$, these collocation points, are chosen to be the images of the Greville abscissas of the associated knot vectors (see §3.4). As an illustration, Fig. 5 depicts the distribution of the Greville collocation points on the Wigley hull using the original knot vectors (upper-left figure) and after inserting 2 (upper right figure) and 4 (lower-middle figure) knots per parametric interval.

Next, we introduce the so-called "induced velocity factors" $\mathbf{u}_{\mathbf{i}}^{q}\left(P_{\mathbf{j}}^{p}\right)$ at the collocation points $P_{\mathbf{j}}^{p}$, namely the "velocity" at $P_{\mathbf{j}}^{p}$ of patch $p$, which is induced by a source-sink distribution (single layer potential) of density equal to NURBS basis function $R_{\mathbf{i}, 1^{q}}^{q}\left(t_{1}, t_{2}\right)$. More accurately,

$\mathbf{u}_{\mathbf{i}}^{q}\left(P_{\mathbf{j}}^{p}\right)=\int_{\Omega_{\mathbf{i}}^{q}} R_{\mathbf{i} \mathbf{1}^{q}}^{q}\left(t_{1}, t_{2}\right)\left[\nabla_{P} G\left(P_{\mathbf{j}}^{p}, \mathbf{x}\left(t_{1}, t_{2}\right)\right)\right] \sqrt{\alpha\left(t_{1}, t_{2}\right)} d t_{1} d t_{2}, \quad p, q=1,2, \ldots, N$ 
where $a=\left\|\mathbf{e}_{1} \times \mathbf{e}_{2}\right\|^{2}$ is the metric tensor determinant, defined through the covariant base vectors, $\mathbf{e}_{1}=\partial \mathbf{x} / \partial t_{1}, \quad \mathbf{e}_{2}=\partial \mathbf{x} / \partial t_{2}, \quad$ and $\quad \Omega_{\mathbf{i}}^{\mathbf{q}}=\operatorname{supp}\left\{R_{\mathbf{i}, l^{q}}^{q}\right\}$ denotes the support of the $R_{\mathrm{i}, \mathrm{I}^{q}}^{q}\left(t_{1}, t_{2}\right)$ contained in $I_{1}^{q} \times I_{2}^{q}$. In the case where the image of the $\Omega_{\mathrm{i}}^{\mathrm{q}}$ has an edge on the undisturbed free-surface plane $(z=0)$, the above equation is modified by including a waterline integral, as follows

$$
\begin{aligned}
\mathbf{u}_{\mathbf{i}}^{q}\left(P_{\mathbf{j}}^{p}\right)= & \int_{\Omega_{\mathbf{i}}^{q}} R_{\mathbf{i}, l^{q}}^{q}\left(t_{1}, t_{2}\right)\left[\nabla_{P} G\right] \sqrt{\alpha\left(t_{1}, t_{2}\right)} d t_{1} d t_{2}+ \\
& +\int_{\Omega_{\mathbf{i}}^{q}\left(t_{1}=t_{1}^{E n d}\right)} R_{\mathbf{i}, 1^{q}}^{q}\left(t_{1}, t_{2}\right)\left[\nabla_{P} G^{*}\right] n_{x}\left(t_{2}\right) \tau_{y}\left(t_{2}\right) \sqrt{\beta\left(t_{2}\right)} d t_{2},
\end{aligned}
$$

where $\beta\left(t_{2}\right)=\left\|\mathbf{e}_{2}\right\|^{2}$ and $G^{*}$ is the regular part of the NK-Green's function (see Eq. $4 \mathrm{~b}$ ). Since $R_{\mathbf{i}, 1^{q}}^{q}\left(t_{1}, t_{2}\right)$ is Hölder continuous, actually it is considerably smoother lying in $C^{k_{1}^{q}-2}\left(I_{1}^{q}\right) \times C^{k_{1}^{q}-2}\left(I_{2}^{q}\right)$, the above integrals exist everywhere except when $p=q$, i.e., if $P_{\mathrm{j}}^{q} \in \Omega_{\mathrm{i}}^{\mathbf{q}}$, in which case they exist as Cauchy Principal Value (PV) integrals (see, e.g., Mikhlin et al 1965). This result breaks down at collocation points $P_{\mathbf{j}}^{q}$ that are images of parametric points lying on the boundary $\partial \Omega_{\mathrm{i}}^{\mathrm{q}}$ of the support $\Omega_{\mathrm{i}}^{\mathrm{q}}$ of the NURBS basis functions $R_{\mathrm{i}, 1^{q}}^{q}\left(t_{1}, t_{2}\right)$, as a result of the occurrence of multiple knots in the initial knot vectors. To circumvent this problem, the present implementation slightly shifts the preimages of such collocation points to the interior of the basis-function support.

\subsection{Integration of singular kernels}

The NK-Green's function $G(P, Q)$, defined in Eq. (4b), can be decomposed into a nonsingular and a singular part, as follows 
$G(P, Q)=G_{\text {sing }}(P, Q)+G_{n o n s i n g}(P, Q), \quad P, Q \in S$,

where

$4 \pi G_{\text {nonsing }}(P, Q)=\left(-R^{-1}+G^{*}(P, Q)\right), \quad G_{\text {sing }}(P, Q)=(4 \pi r)^{-1}, r=|P Q|, R=\left|P Q^{\prime}\right|$,

with $Q^{\prime}$ denoting the image of $Q$ with respect to the undisturbed free surface $\mathrm{z}=0$. For the calculation of the non-singular part $\left(G^{*}\right)$ of the Green's function, which involves zexponential decaying and wavelike components, a procedure based on Newman (1987a,b) analysis is followed. However, the series expansion associated with the wavelike component becomes unstable as the points $P, Q$ approach the $z=0$ plane, leading to numerical instabilities, particularly as concerns $G^{*}$ derivatives; see also the discussion by Marr \& Jackson (1999). At present, this problem is numerically treated by vertically downshifting the whole hull by a small parameter $\delta z=\lambda / \alpha$ of the order of wavelength $\lambda=2 \pi / k$ of the transverse component of the wave system generated by the moving ship, where $\alpha$ is a factor. The optimum value of the latter factor depends on ship geometry and can be specified by numerical experimentation; see Sec. 4.5 , where the case of a series 60 ship hull is treated. Future work will be focusing on optimizing the calculation of $G^{*}$, as described in ibid. Obviously, no such numerical correction is needed in the case of fully submerged bodies. To proceed, in accordance with Eq. (10a), the integral on $\Omega_{i}^{q}$ in Eqs. (9a) and (9b) is split to two parts as follows

$\mathbf{u}_{\mathbf{i}}\left(P_{\mathbf{j}}^{p}\right)=\mathbf{u}_{\mathbf{i}}^{(\text {nonsing })}\left(P_{\mathbf{j}}^{p}\right)+\mathbf{u}_{\mathbf{i}}^{(\text {sing })}\left(P_{\mathbf{j}}^{p}\right)$.

The integration of the non-singular part $\mathbf{u}_{\mathbf{i}}^{(\text {nonsing })}\left(P_{\mathbf{j}}^{p}\right)$ is easily obtained by using standard numerical quadrature rules. In the present version, Gauss-Kronrod quadrature formulas are implemented using points and weights ranging from 21 to 51; see, e.g., Press et al (1992), 
Shampine (2008). For the evaluation of the singular part $\mathbf{u}_{\mathbf{i}}^{(\text {sing })}\left(P_{\mathbf{j}}^{p}\right)$ we distinguish three cases as follows:

(i) far-field case: The preimage of $P_{\mathbf{j}}^{p}$ does not lie in $\Omega_{\mathrm{i}}^{\mathrm{q}}$ and its Euclidean distance $d\left(\mathbf{P}_{j}^{p}, \operatorname{conv}_{i}^{q}\right)$ from the convex hull $\operatorname{conv}_{i}^{q}$ of the control net of $\mathbf{x}\left(t_{1}, t_{2}\right),\left(\mathbf{t}_{\mathbf{1}}, \mathbf{t}_{\mathbf{2}}\right) \in \Omega_{\mathbf{i}}^{\mathbf{q}}$ is greater than twice its diameter ${ }^{(1)} \operatorname{diam}\left(\operatorname{con} v_{i}^{q}\right)$. In this case, the integrand behaves regularly and the same, as above, 21-51 points Gauss-Kronrod quadrature formulae are applied.

(ii) near-field case: The preimage of $P_{\mathbf{j}}^{p}$ remains outside $\Omega_{\mathbf{i}}{ }^{q}$, but $d\left(\mathbf{P}_{j}^{p}, \operatorname{con} v_{i}^{q}\right)<2 \operatorname{diam}\left(\operatorname{con} v_{i}^{q}\right)$, in which case numerical experience has shown that numerical instabilities could occasionally appear. In the present work an alternative scheme for the evaluation of the integral in this case is used, based on transformation techniques permitting the numerical grid (used for the integration) to become finer at the integration points $Q$ which are closer to $P_{\mathbf{j}}^{p}$; see, e.g., Telles (1987), Telles \& Oliveira (1994) and Voutsinas \& Bergeles (1992).

(iii) in-field case: The preimage of $P_{\mathrm{j}}^{p}$ lies in the interior of $\Omega_{\mathrm{i}}^{\mathrm{q}}$, in which case the kernel is singular and the integral should be considered in the Cauchy-PV sense, see, e.g., Mikhlin (1965). For the evaluation of the Cauchy-PV, an $\varepsilon$-neighborhood, cutting-off of the singularity at $P_{\mathbf{j}}^{p}$, is introduced and subsequently the limit as $\varepsilon \rightarrow 0$ is calculated; see, e.g., Mikhlin (1965). An important aspect concerning the implementation of the discrete Cauchy-PV is that the discretization parameter $h$ (equivalent mesh size for the numerical 
integration) should depend on the cut-off parameter $\varepsilon$ so that $h_{\varepsilon} \rightarrow 0$ as $\varepsilon \rightarrow 0$. More details concerning the treatment of the singular integrals and the achieved rates of convergence are provided in the Appendix.

\subsection{The discrete scheme}

On the basis of the assumptions made in the previous subsections, namely that the wetted hull surface $S$ is a multi-patch NURBS surface represented as in Eqs. (7) and that the unknown source-sink distribution $\mu$ on $S$ is embedded in the spline space $\mathbb{S}_{1}^{p}$ by inheriting the NURBS basis used for representing $S$ (see Eq. 8), the projection $\Pi$ of the integrals in the left hand side of Eq. (5) on $\mathbb{S}_{1}^{p}$ is materialized by evaluating them on selected collocation points $P_{\mathbf{j}}^{p} \in S$, namely,

$$
\Pi\left(P_{\mathbf{j}}^{p}\right)=\int_{S} \mu(Q) \frac{\partial G\left(P_{\mathbf{j}}^{p}, Q\right)}{\partial n\left(P_{\mathbf{j}}^{p}\right)} d S(Q)+\frac{1}{k} \int_{\ell} \mu(Q) \frac{\partial G^{*}\left(P_{\mathbf{j}}^{p}, Q\right)}{\partial n\left(P_{\mathbf{j}}^{p}\right)} n_{x}(Q) \tau_{y}(Q) d \ell(Q),
$$

which, after some straightforward calculus, takes the following form:

$$
\Pi\left(P_{\mathbf{j}}^{p}\right)=\sum_{q=1}^{N} \sum_{\mathbf{i}=0}^{\mathbf{n}^{q}+\mathbf{l}^{q}} \mu_{\mathbf{i}}^{q}\left[\mathbf{u}_{\mathbf{i}}^{q}\left(P_{\mathbf{j}}^{p}\right) \cdot \mathbf{n}\left(P_{\mathbf{j}}^{p}\right)\right]
$$

where $\mathbf{u}_{\mathbf{i}}^{q}\left(P_{\mathbf{j}}^{p}\right), P_{\mathbf{j}}^{p} \in S$, are the induced velocity factors defined by Eqs. (9) and $\mathbf{n}=\mathbf{e}_{1} \times \mathbf{e}_{2} / \sqrt{a}$ is the outward unit normal vector on $S$. Using (12b) the projection of the BIE (5) on $\mathbb{S}_{1}^{p}$ takes the form of a linear system with respect to the unknown coefficients $\left\{\mu_{\mathrm{i}}^{p}\right\}$ as below:

(1) The convex hull for a set of points $X$ is the minimal convex set containing $X$. In our case $X$ is the set of control vertices of $\mathbf{x}\left(t_{1}, t_{2}\right),\left(\mathbf{t}_{\mathbf{1}}, \mathbf{t}_{\mathbf{2}}\right) \in \Omega_{\mathbf{i}}^{\mathbf{q}}$ and, as a result, $\operatorname{conv}_{i}^{q}$ is a convex polytope. The diameter $\operatorname{diam}\left(\operatorname{conv}_{i}^{q}\right)$ of $\operatorname{con} v_{i}^{q}$ is the maximum distance over all pairs of vertices of $\operatorname{con} v_{i}^{q}$. 


$$
\sum_{\mathbf{i}=0}^{\mathbf{n}^{p}+\mathbf{l}^{p}} \mu_{\mathbf{i}}^{p} R_{\mathbf{i}, \mathbf{l}^{p}}^{p}\left(t_{1, j_{1}}^{p}, t_{2, j_{2}}^{p}\right)-2 \Pi\left(P_{\mathbf{j}}^{p}\right)=-2 \mathbf{U}\left(P_{\mathbf{j}}^{p}\right) \cdot \mathbf{n}\left(P_{\mathbf{j}}^{p}\right), \quad \mathbf{j}=0, \ldots ., \mathbf{n}^{p}+\mathbf{l}^{p}, p=1, \ldots N
$$

In order to ensure the solvability of the linear system (13), we select the collocation points to be the Greville abscissas of the associated NURBS bases; see, e.g., Farin (2001). As noted previously, since some knots (including boundary knots) in initial knot vectors are usually multiple, it is likely to arise that Greville abscissas lie to the boundary of the support of the NURBS bases, which, in turn, renders problematic the evaluation of integrals with Cauchy singularity. To overcome this difficulty, these collocation points are slightly shifted from the boundary inside the basis-functions support, in such a way so that the symmetry of the arrangement of the preimages of the collocation points is maintained, as far as possible. More details concerning the effect of this shifting on the convergence rate are provided at the end of Sec.4.1.

Convergence of the solution of the discrete BIE (13) to the solution of the continuous BIE (5) is materialized through h-refinement, consisting in enriching the family of collocation points through knot insertion. Alternative mechanisms involve degree elevation ( $p$ refinement) or combing degree elevation with knot insertion (k-refinement); see, e.g., Politis et al (2009). As an example, Fig. 5 depicts two levels (Figs. 5b,c) of enriching the initial collocation net (Fig. 5a) for the Wigley hull through knot insertion.

After solving the linear system (13), induced velocities at the collocation points are calculated using the induced velocity factors $\mathbf{u}_{\mathbf{i}}^{q}\left(P_{\mathbf{j}}^{p}\right)$, defined by Eqs.(9), as follows

$$
\mathbf{u}\left(P_{\mathbf{j}}^{p}\right)=\sum_{q=1}^{N} \sum_{\mathbf{i}=0}^{\mathbf{n}^{q}+1^{q}} \mu_{\mathbf{i}}^{q}\left(\frac{1}{2} R_{\mathbf{i}, 1^{q}}^{q}\left(t_{1, j_{1}}^{p}, t_{2, j_{2}}^{p}\right) \mathbf{n}\left(P_{\mathbf{j}}^{p}\right)+\mathbf{u}_{\mathbf{i}}^{q}\left(P_{\mathbf{j}}^{p}\right)\right), \mathbf{j}=0, \ldots ., \mathbf{n}^{p}+\mathbf{l}^{p}, p=1, \ldots N,(14 \mathrm{a})
$$

including $\mu \mathbf{n} / 2$ as the first term in the right-hand side, from the jump relations of the single layer potential (see, e.g., Günter 1967, Kress 1989). Moreover, total velocities at the collocation points are obtained from Eq.(4) as follows 
$\mathbf{v}\left(P_{\mathbf{j}}^{p}\right)=\mathbf{U}+\mathbf{u}\left(P_{\mathbf{j}}^{p}\right)$

Also, free-surface elevation is calculated from the $x$-component of the disturbance velocity on $z=0$ using Eq.(6b), and pressure distribution on the hull surface is obtained by Eq. (6a). Finally, forces and moments are calculated by means of pressure integration on $S$, combined with the appropriate components of the normal vector and other geometrical quantities.

\section{NUMERICAL RESULTS AND DISCUSSION}

In order to test the accuracy and robustness of the IGA-BEM method presented in $\S 3$, this section provides a series of numerical results for fully submerged and surface piercing bodies in a variety of physical configurations.

\subsection{A prolate spheroid in infinite domain}

As a starting example, we consider a 3-axial ellipsoid with axes ratio 5:1:1 (prolate spheroid), moving at constant speed in infinite homogeneous fluid. In this case, an analytical solution is available (see, e.g., Lamb 1932 or Milne-Thomson 1968) supporting the calculation of absolute error and rate of convergence of the present numerical solution. In our study the $\mathrm{L}^{2}$-error associated with the velocity field on the body surface is defined as follows:

$\left\|\mathbf{v}-\mathbf{v}^{M}\right\|=\left(\sum_{\ell=1}^{3} \sum_{p=1}^{N} \int_{I_{1}^{p} \times I_{2}^{p}}\left|v_{\ell}-v_{\ell}^{M}\right|^{2} \sqrt{a} d t_{1} d t_{2}\right)^{1 / 2}$,

where $a\left(t_{1}, t_{2}\right)$ is the metric-tensor determinant of the ellipsoid's surface, $\mathbf{v}=\left(v_{1}, v_{2}, v_{3}\right)=\left(v_{x}, v_{y}, v_{z}\right)$ is the total velocity vector field on the surface of the body (see 
Eq. 4) and $\mathbf{v}^{M}$ denotes its IGA-BEM approximation based on a particular approximation characterized by the total number $M$ of degrees of freedom. Finally, the quantities $\left|v_{\ell}-v_{\ell}^{M}\right|, \ell=1,2,3$, represent the local error of each velocity component, respectively.

The distribution of the $x$-component of the velocity along a meridian connecting the geometrical poles of the above ellipsoid, for an inflow parallel to its great axis, is shown in Fig. 6, where it is compared against the analytical solution. In particular, results obtained by using two different single-patch parameterizations of the ellipsoid based on rational quadratic NURBS are shown in Fig.6. In the first representation, named $x$-axis parameterization, the poles are located at the points $\mathbf{x} / L=( \pm 0.5,0,0)$, where $L$ is the length of the ellipsoid, and coincide with the stagnation points of the flow. Thus, in this case, the $t_{1}$ isoparametrics correspond to meridians. In the second representation, named $z$ axis parameterization, the poles are located at $\mathbf{x} / L=(0,0, \pm 0.1)$.

In both cases the number of dof increases in proportionality to the product of knots inserted along each parametric direction $t_{1}$ and $t_{2}$. We observe in Fig.6 that as the number of additional knots increases ( $h$-refinement) the error diminishes rapidly, except in the vicinity of the stagnation points (see Fig. 6a), which is more-or-less expected due to the large variation of the solution in this region. The situation improves when using the $z$ parameterization of the ellipsoid, in which case the poles of the geometry representation do not coincide with the stagnation points; see Fig. 6b. In order to further improve convergence, we could use graded parameterizations, leading to local refinement of distributions of Greville (collocation) points near the stagnation points, or multi-patch representations for removing the poles. 
The $\mathrm{L}^{2}$-error (Eq. 15), as obtained using the $z$-parameterization, is plotted in Fig.7(a) against the number of dof. In order to approximate the integral in (Eq. 15) a trapezoidal rule has been applied, based on data at the collocation points with variable grid size. In the same figure the corresponding result obtained by raising the NURBS degree from 2 to 3 and 4 ( $k$-refinement) is also plotted. Results obtained by the low-order panel method, based on quadrilateral boundary elements carrying constant source-sink density, are also including in the same figure using a black solid line. In this example, meaningful results by the low-order panel method are obtained using discretizations larger than 200 elements. In order to better illustrate the behaviour of the low-order panel method, an artificial extension of the corresponding error line below 200 dof is included in Fig.7(a) by using a dashed black line.

We observe that the convergence rate associated with the proposed IGA-BEM method, is several orders of magnitude higher than the low-order panel method. For example, we see in the above figure that for an $\mathrm{L}^{2}$-error level of $2 \%$, the present method requires less than 200 dof depending on the NURBS degree, while the low-order panel method necessitates more than 1200 panels, and this difference rapidly increases for lower error levels. Moreover, we observe in Fig.7 that our method presents a tendency for exponential convergence, as indicated by the continuous increase of the downslope of the error curve measured in the logarithmic scale, in contrast to the low-order panel method, which exhibits a slow algebraic convergence. Similar conclusions have been drawn by studying two dimensional boundary value problems governed by the Laplace equation; see Politis et al (2009).

As usual in BEM, the $\mathrm{L}^{2}$-error is affected by several parameters. The first and most important one deals with the discretization (number of dof), which reflects the accuracy of the approximation of the sought for solution by its projection in the finite dimensional 
NURBS space $\mathbb{S}_{1}^{p}$; see $\S 3.2$. A second significant parameter deals with the accuracy of calculation of the different types of integrals (singular and/or regular) involved in the BIE, which is usually set to a fixed value. Therefore, as dof increases, after a threshold depending on the rate of convergence, the numerical-integration error dominates and the

$\mathrm{L}^{2}$-error cannot be further improved, leading to a plateau behaviour, as indicated by the thick dashed-dot line in Fig.7(a). Of course, this limit (plateau) can be lowered, by increasing the accuracy of numerical integration, as illustrated in Fig. 7(b), where convergence rates for NURBS degrees 3 and 4 are presented for two choices of integration parameters, specifically, for 1501 and 3501 points respectively, for the calculation of singular integrals. Also, the effect of slight shifting the boundary collocation points by a small parameter $(\varepsilon)$ on the rate of convergence is illustrated in Figure 7(c). We clearly observe that, as dof increases, the convergence characteristics of the present scheme are not affected, with $\varepsilon$ in the interval $0.001<\varepsilon<0.05$.

\subsection{A rapidly moving submerged ellipsoid}

As a next example, we consider a 3-axial, totally submerged ellipsoid, with axes ratio 2:1:0.5, moving at constant speed in semi-infinite domain, which is bounded above by a horizontal plane, where a homogeneous Dirichlet condition is applied. This problem asymptotically models the linearized solution corresponding to flow around a rapidly moving body under the free surface and its Green function comprises only the first two terms in (Eq. 4b), i.e., the Rankine source term and its image with respect to the $z=0$ plane. Fig. 8 depicts the horizontal velocity distribution (left Fig. 8) along the top meridian on the $x z$-plane of the above ellipsoid at low submergence $d / L=0.16$, with $L$ denoting the length of the ellipsoid, and the velocity vector field over the surface (right Fig. 8). 
The present results have been obtained using the $x$-axis parameterization of the 3D ellipsoid after inserting 2, 4 and 8 knots per parametric interval, which results in 153, 325 and 861 dof, respectively. In this case analytical solution is not available and thus, convergence of the numerical solution is estimated using the $\mathrm{L}^{2}$ relative error, defined as follows:

$$
\left\|\mathbf{v}^{M}-\mathbf{v}^{M-K}\right\|=\left(\sum_{\ell=1}^{3} \sum_{p=1}^{N} \int_{I_{1}^{P} \times I_{2}^{p}}\left|v_{\ell}^{M}-v_{\ell}^{M-K}\right|^{2} \sqrt{a} d t_{1} d t_{2}\right)^{1 / 2},
$$

with $K$ a fixed index, $K<M$.

\subsection{A submerged prolate spheroid under the free-surface}

In this subsection results are presented concerning the solution of the linear NK wavemaking problem for the prolate spheroid of $\S 4.1$, translating steadily at low submergence $(d / L=0.16)$ with Froude number $F=U / \sqrt{g L}=0.5$. Reference solutions of this problem are available; see, e.g., Farell (1973), Doctors \& Beck (1987). The resulting wave pattern is depicted in Fig. 9. The wave resistance $R_{W}$ at various speeds is obtained by pressure integration on the surface of the submerged body, and the corresponding wave-resistance coefficient $C_{W}$ is calculated by means of the following equation

$$
C_{W}=\frac{R_{W}}{0.5 \rho U^{2} S_{W}}=S_{W}^{-1} \sum_{p=1}^{N}\left(\int_{I_{1}^{p} \times I_{2}^{p}} C_{P} n_{x} \sqrt{a} d t_{1} d t_{2}\right),
$$

where $S_{W}$ denotes the area of the wetted surface $S$ of the body, $n_{x}$ is the $x$-component of the unit-normal vector on $S$ and $C_{P}$ stands for the pressure coefficient defined as:

$$
C_{p}=\frac{p-p_{\infty}}{0.5 \rho U^{2}}=1-(v / U)^{2}-2 g z / U^{2},
$$


with $v=\|\mathbf{v}\|=\|\mathbf{U}+\nabla \varphi\|$ denoting the total flow velocity. Other important coefficients, as those concerning sinkage and trim, can be calculated by similar formulas. The pressure coefficient $C_{P}$ along a series of meridians of the prolate spheroid, as obtained by the present method using $M=325$ dof and Eq. (18), is shown in Fig. 10.

In Fig. 11 the wave resistance coefficient $C_{W}$ of the prolate spheroid at low submergence $d / L=0.16$ is given for seven Froude numbers, as calculated by Eq. (17) and again $M=325$ dof. We observe that the predictions obtained by the present method converge rapidly to the reference solution by Doctors \& Beck (1987), shown in the same figure by using a thick line. It is clear that the present IGA results, obtained by inserting 2 and 4 knots per parametric interval, which correspond to $M=135$ and 325 dof, respectively, are much more accurate than the ones (depicted by crosses) obtained by the low-order panel method using a grid of $16 \times 20$ elements (on the surface of the whole body), which corresponds to 320 dof.

\subsection{A Wigley parabolic hull}

In this subsection the performance of the proposed method for a surface-piercing hull is presented and discussed. The hull in question is the standard parabolic Wigley parabolic hull (Sec.3.1), with main-dimension ratios: $L / B=10, L / T=16$ and $B / T=1.6$.

In particular, various single- and multi-patch representations of this hull have been used in order to test the applicability and convergence of the IGA-BEM approach in the case of surface piercing bodies moving at constant forward speed. As an illustration, we present in Fig. 12 numerical results concerning the calculated source-sink distribution $\mu$ on the surface of the hull, as obtained by using the single-patch representation of the Wigley hull and refinement levels 1 and 4. Apparently, $\mu$ converged already at refinement level 1 . In order to test the multi-patch version of the code, artificial multi-patch NURBS 
representations of the Wigley hull have been generated by subdividing the single-patch surface along specific isoparametrics. Selected results obtained by using two vertical, two horizontal, and four patches are illustrated in Fig.13. We clearly observe in this figure that the colour plots of the computed source-sink distributions apparently coincide (mean difference, with respect to single-patch surface, less than $1.2 \%$ ) for all three alternative multi-patch representations at the same refinement level (4), which ensures the robustness of the proposed method. However, in the case of more complex, realistic ship hulls, different patch representations could give rise to different performance, implying the necessity of a preliminary study in order to achieve optimum surface representation.

It is worth mentioning here that first comparisons between bi-quadratic isogeometric NURBS and bi-quadratic Lagrange elements reveal similar rates of convergence. However, for the same dof, the present IGA-BEM exhibits better performance, as for example illustrated in Fig.14 concerning the Wigley hull. Similar behavior has been also recently reported by Li \& Qian (2011). This is due to the fact that in the case of bi-quadratic NURBS a $\mathrm{C}^{1}$ inter-element continuity of the sought for solution is obtained, while in the case of Lagrange elements the (global) continuity is only $\mathrm{C}^{0}$. In addition, it is also to be noted that in the present method the boundary surface is treated exactly (as it is produced by the CAD software), while in other approaches, based either on low or high order BEM, an additional error is introduced by the approximation of the geometry.

Using the single patch representation, the calculated wave pattern of the Wigley hull, steadily advancing at Froude number $F=0.316$, is shown in Fig. 15. In addition, color plots of the pressure distributions on the hull surface for Froude numbers $F=0.267$ and $F=0.316$, are presented in Fig. 16, respectively, as obtained by the present method, using $N=703$ dof (refinement level 4). For the latter Froude number, predictions of the wave profile alongside the Wigley hull are compared in Fig. 17 against results by other methods (Maskew et al, 1997) and experimental data. Results of the present method are depicted 
in red, while results from the USAERO code (ibid.) are denoted by thin solid lines connecting hollow triangles or circles, and, finally, experimental data are depicted by disconnected hollow circles. Fig. 17 implies that our results agree relatively well with theoretical predictions and measured data over the whole length of the ship with the exception of an area near the stern, where the present method overestimates the wave profile. Finally, in Fig. 18, the wave resistance coefficient $C_{W}$ of the Wigley hull is depicted in red for various Froude numbers, as calculated by the present IGA method and $M=703$ dof. We observe that our predictions lie within the range of experimental data provided by ITTC (1987) (see also Nakos \& Sclavounos 1994) and agree well with predictions by other BEM, as, e.g., those provided recently by Bal (2008). As before, in comparison with the low-order panel method, the present method guarantees better accuracy for the same dof.

We conclude this subsection by utilizing the above Wigley hull to provide additional information concerning the efficiency of the present IGA-BEM. Fig. 19 depicts computation time versus dof for a computer cluster, with $1+8$ (front end + computing) nodes, each one with 2 Xeon Quad CPUs@2.4MHz and 12Gb memory, connected through 10GBit network, and storage capacity of $4 \mathrm{~Tb}$. We observe that the computation cost increases at least quadratically with dof. Based on this and similar experimentations we consider that the present method is applicable for systematic calculations involved in hull optimization problems, provided that accurate solutions are obtained by using a total number of dof of $O\left(10^{3}\right)$. First results in this direction have been presented in Ginnis et al (2011).

\subsection{A Series 60 ship hull}

As a final example, numerical results are presented in this subsection concerning the application of the IGA-BEM method to the calculation of the wave field and wave 
resistance of a ship hull belonging to Series 60 , with block coefficient $\mathrm{Cb}=0.60$. Extensive experimental and theoretical results concerning this hull model are available from $18^{\text {th }}$ ITTC (1987); see also Nakos \& Sclavounos (1994), Mori et al (1994).

The main dimension ratios of the above Series 60 hull are: $L / B=7.5, L / T=18.75$, $B / T=2.5$. A multipatch representation was developed to treat this classical round stern hull, composed by 7 patches, as previously discussed in Sec.3.1 and illustrated in Fig.4. In particular, the calculated source-sink distribution $\mu$ on the hull surface, for Froude number $F=0.316$, is presented in Fig. 20, as obtained by the present method with $M=3285$ dof, which is found to be enough for convergence. The above surface plot has been obtained using the calculated values of $\mu$ at the collocation points. The corresponding surface velocities and distribution of the pressure coefficient on the hull surface (for the same as before Froude number) are shown in Fig. 21 and 22, respectively. In particular, in Fig. 22 we observe the rapid increase of the pressure at the stern area of the above hull which is responsible for the relative decrease of the wave resistance in this regime of ship speeds.

Finally, in Fig. 23 the wave resistance coefficient $C_{W}$ of the examined Series 60 hull is depicted for various Froude numbers, as calculated by the present IGA method and using $M=3285$ dof. Also, in the same figure, the effect of artificial sinkage $(\delta z)$ introduced for the numerical treatment of the $G^{*}$-part of the Green's function and its derivatives is illustrated. In particular, three values of $\delta z / \lambda=\alpha^{-1}=1 / 20,1 / 25,1 / 30$ are shown by using green, blue and red lines, respectively. We conclude from this and many similar studies that reasonable predictions of the wave resistance are obtained for Froude numbers up to 0.35 , which is considered to be extremely high for this type of fulldisplacement ship hulls. Moreover, we observe in this example that our predictions based on $\alpha=25 \div 30$, are in good agreement with other well-known and established methods, 
as the BEM developed by Nakos \& Sclavounos (1994) using their calculations obtained by pressure integration on the hull surface. Finally, present method results are found to be in conformity with experimental data provided by ITTC (1987) survey, especially for higher values of the Froude number.

\section{CONCLUSIONS}

In the present work IGA, initially proposed by Hughes et al (2005), is applied for solving the BIE associated with the NK problem and the calculation of the wave resistance of ships and steadily translated submerged bodies. The isogeometric concept is based on the exploitation of the same NURBS basis for exactly representing the body geometry and for approximating, through refinement process, the singularity distribution of the associated BIE or, in general, the dependent physical quantities. To this respect, the present approach, although focusing on the linear NK problem, exhibits the novelty of providing a paradigm of integrating contemporary CAD systems for ship-hull design, that rely nowadays almost exclusively on NURBS, with CFD solvers. The enhanced accuracy and efficiency of the present method has been demonstrated by comparing numerical results obtained in the case of a prolate spheroid in infinite domain, a three-axial ellipsoid in semi-infinite domain, a submerged prolate spheroid under the free surface, and two surface piercing hulls, the standard Wigley parabolic hull and a Series-60 $\left(\mathrm{C}_{\mathrm{b}}=0.60\right)$ hull, against analytical solution, experimental data and predictions provided by low-order panel methods and other established BEM from the pertinent literature. Future work is planned towards the detailed analysis of rates of convergence of the present method and its exploitation to the optimization of ship hulls with respect to wave resistance. 


\section{ACKNOWLEDGMENTS}

Financial support by the FP7 EC-Project: Exact Geometry Simulation for Optimized Design of Vehicles and Vessels (EXCITING, SCP8-2007-GA-218536) is gratefully acknowledged. Also, the authors would like to thank Dr A. Theodoulides from HRS (also an EXCITING partner) for providing access to a software implementation of the NKGreen's function and Mr J. Karigiannis for helping with the computations.

\section{APPENDIX. Treatment of the singular integrals}

In the in-field case, where the preimage of collocation point $P_{\mathbf{j}}^{p}$ lies in the interior of $\Omega_{\mathbf{i}}^{\mathbf{q}}\left(P_{\mathbf{j}}^{q} \in \Omega_{\mathbf{i}}^{\mathbf{q}}\right)$, the 2D singular integral, Eqs.(9), is written in the form

$$
\mathbf{u}_{\mathbf{i}}^{q}\left(P_{\mathbf{j}}^{p}\right)=\int_{t_{2}^{A}}^{t_{2}^{B} \int_{1}^{A}} \int_{1}^{t_{1}^{B}} \mathbf{f}\left(t_{1}, t_{2}\right) d t_{1} d t_{2},
$$

where $\mathbf{f}\left(t_{1}, t_{2}\right)=R_{\mathbf{i}, 1^{q}}^{q}\left(t_{1}, t_{2}\right)\left[\nabla_{P} G\right] \sqrt{\alpha\left(t_{1}, t_{2}\right)}, \quad \Omega_{\mathbf{i}}^{\mathbf{q}}=\left[t_{1}^{A}, t_{1}^{B}\right] \times\left[t_{2}^{A}, t_{2}^{B}\right]$, and $P_{\mathbf{j}}^{p} \quad$ is the image of $\left(t_{1}^{P}, t_{2}^{P}\right)$ on the parameter space; see Fig. A.1. The integral in (A.1) is defined as a Cauchy principal-value one, and for its numerical calculation an $\varepsilon$ - neighborhood is introduced, cutting-off the singularity, obtaining

$$
\begin{aligned}
\mathbf{u}_{\mathbf{i}}^{q}\left(P_{\mathbf{j}}^{p}\right)=\lim _{\varepsilon \rightarrow 0}\left\{\int_{t_{2}^{A}}^{t_{2}^{P}-\varepsilon} \int_{t_{1}^{A}}^{t_{1}^{B}} \mathbf{f}\left(t_{1}, t_{2}\right) d t_{1} d t_{2}\right. & +\int_{t_{2}^{P}+\varepsilon}^{t_{2}^{B}} \int_{t_{1}^{A}}^{t_{1}^{B}} \mathbf{f}\left(t_{1}, t_{2}\right) d t_{1} d t_{2}+ \\
& \left.+\int_{t_{2}^{P}-\varepsilon}^{t_{2}^{P}+\varepsilon} \int_{t_{1}^{A}}^{t_{1}^{P}-\varepsilon} \mathbf{f}\left(t_{1}, t_{2}\right) d t_{1} d t_{2}+\int_{t_{2}^{P}-\varepsilon}^{t_{2}^{P}+\varepsilon} \int_{t_{1}^{P}+\varepsilon}^{t_{1}^{B}} \mathbf{f}\left(t_{1}, t_{2}\right) d t_{1} d t_{2}\right\} .
\end{aligned}
$$

The domains of the above four integrals are denoted in Fig.A.1 as I, II, III and IV, respectively. All these integrals are nearly singular (in the sense that the singularity is outside the corresponding domain of integration), and thus, the method introduced by Telles (1987) is used as described in the sequel. By linear transformation of the involved variables, each one of the above integrals is put in the following form 
$\mathbf{J}=\int_{-1}^{1} \int_{-1}^{1} \mathbf{f}_{*}\left(\xi_{1}, \xi_{2}\right) d \xi_{1} d \xi_{2}, \quad$ where $\quad \mathbf{f}_{*}=|d \mathbf{t} / d \xi| \mathbf{f}$

and $\mathbf{f}_{*}\left(\xi_{1}, \xi_{2}\right)$ is singular at the point $\left(\xi_{1}^{P}, \xi_{2}^{P}\right)=\left(\xi_{1}\left(t_{1}^{P}, t_{2}^{P}\right), \xi_{2}\left(t_{1}^{P}, t_{2}^{P}\right)\right)$. Following Telles \& Oliveira (1994), we introduce now the non-linear transformation(s)

$\xi_{k}\left(s_{k}\right)=a_{k} s_{k}^{3}+b_{k} s_{k}^{2}+c_{k} s_{k}+d_{k}, \quad k=1,2$

where the coefficients $a_{k}, b_{k}, c_{k}, d_{k}$, are calculated such that

$\xi_{k}\left(s_{k}=-1\right)=-1, \quad \xi_{k}\left(s_{k}=1\right)=1, \frac{d \xi_{k}\left(s_{k}=s_{k}^{P}\right)}{d s_{k}}=0, \frac{d^{2} \xi_{k}\left(s_{k}=s_{k}^{P}\right)}{d s_{k}^{2}}=0, \quad k=1,2$,

and $\left(s_{1}^{P}, s_{2}^{P}\right)$ denotes the point of singularity, i.e. $\xi_{k}^{P}=\xi_{k}\left(s_{k}^{P}\right), k=1,2$. Then the coefficients of the transformation (A.4) are obtained as

$a_{k}=Q_{k}, b_{k}=-3 s_{k}^{P} Q_{k}, c_{k}=3\left(s_{k}^{P}\right)^{2} Q_{k}, d_{k}=-b_{k}$, and $Q_{k}^{-1}=1+3\left(s_{k}^{P}\right)^{2}, k=1,2$.

Consequently, the integrals (A.3) are reduced to the following form

$\mathbf{J}=\int_{-1}^{1} \int_{-1}^{1} \mathbf{f}_{*}\left(\xi_{1}\left(s_{1}\right), \xi_{2}\left(s_{2}\right)\right)\left(9 Q_{1} Q_{2}\left(s_{1}-s_{1}^{P}\right)^{2}\left(s_{2}-s_{2}^{P}\right)^{2}\right) d s_{1} d s_{2}$,

which are calculated by using standard quadrature rules based on $N$ points. The advantage of the above procedure relies to the fact that the non-linear transformations (A.4) produce a lumping effect of integration points as approaching the point of singularity. Extensive numerical evidence from a variety of examples considered has shown that the error decays like $|\mathbf{J}(N)-\mathbf{J}| \approx N^{-m}$, with $m>1$, suggesting that $\mathbf{J}(N)$ exhibits fast rate of convergence.

\section{REFERENCES}

Bal, S., 2008. Prediction of wave pattern and wave resistance of surface piercing bodies by a boundary element method, Int. Journ. for Num. Meth. in Fluids 56 (3), pp. 305-29. 
Baar J. J. M., Price W.G., 1988. Developments in the Calculation of the Wave-making Resistance of Ships, Proc. Royal Society of London. Series A, Mathematical and Physical Sciences 416, No. 1850, 115-147.

Bertram V. 2000. Practical Ship Hydrodynamics, Butterworth-Heinemann, Oxford

Brard, R. 1972. The representation of a given ship form by singularity distributions when the boundary condition on the free surface is linearized, J. Ship Res. 16, 79-82.

Brebbia C., 2002. Recent innovations in BEM. Engineering Analysis with Boundary Elements 26.

Brebbia C., Telles J., and Wrobel L. 1984. Boundary Element Techniques. Springer Verlag, Berlin.

Cottrell J.A., Hughes T.J.R., Reali A., 2007. Studies of refinement and continuity in isogeometric structural analysis, Computer Methods in Applied Mechanics and Engineering, 196, 4160-4183.

Cottrell J.A., Hughes T.J.R., Bazilevs Y., 2009. Isogeometric Analysis: Toward Integration of CAD and FEA, Wiley.

Datta, R., Sen, D., 2006. A B-spline solver for the forward-speed diffraction problem of a floating body in the time domain, Applied Ocean Res. 28, 147-160.

Datta, R., Sen, D., 2007. The simulation of ship motions using a B-spline based panel method, Jour. of Ship Research, 51(3), 267-284.

Dawson C.W., 1977. A practical computer method for solving ship-wave problems, Proc. 2nd Intern. Conference on Numerical Ship Hydrodynamics.

Doctors L. J., Beck R. F. 1987. Convergence properties of the Neumann-Kelvin problem for a submerged body, Journal of Ship Research 31(4), 227-234.

Farell C., 1973. On the wave resistance of submerged spheroid, J . Ship Res. 17, 1-11.

Farin G., 2001. Curves and surfaces for CAGD, a Practical Guide (5th Edition). Morgan Kaufmann Publishers. 
Gao Z., Zou Z., 2008. A NURBS based high-order panel method for three-dimensional radiation and diffraction problems with forward speed. Ocean Engineering 35, 12711282.

Gennaretti M., Calcagno G., Zamboni A., Morino L. 1998. A high order boundary element formulation for potential incompressible aerodynamics, Aeronautical Journal 102, (1014), 211-219.

Ginnis A.I., Kostas K.V., Feurer C., Belibassakis K.A., Gerostathis Th.P., Politis C.G., Kaklis P.D., 2011. A CATIA® ship-parametric model for isogeometric hull optimization with respect to wave resistance, Proc. $15^{\text {th }}$ International Conference on Computer Applications on Shipbuilding, ICCAS 2011, Trieste.

Günter, N.M., 1967. Potential theory and its applications to basic problems of mathematical physics, Fr. Urgan Publ.

Hess J. 1975. Improved solution for potential flow about axisymmetric bodies by use of a higher order surface source method. Comp. Meth. Appl. Mech. Eng. 5, 297-308.

Hughes T.J.R., Cottrell J.A., Bazilevs Y., 2005. Isogeometric analysis: CAD, finite elements, NURBS, exact geometry and mesh refinement. Computer Methods in Applied Mechanics and Engineering 194, 4135-4195.

Hughes T.J.R., 2008. Isogeometric analysis: Progress and Challenges, International Conference on Mathematical Methods for Curves \& Surfaces (MMCS'08) Tromso.

ITTC 1987. Report of the Resistance and Flow Committee, $18^{\text {th }}$ Inter. Towing Tank Conf., Kobe, Japan.

ITTC 2005. Report of the Resistance Committee, $24^{\text {th }}$ Inter. Towing Tank Conf., Edinburgh, U.K.

ITTC 2008. Report of the Resistance Committee, $24^{\text {th }}$ Inter. Towing Tank Conf. Fukuoka, Japan.

Katz, J., Plotkin, 1991. Low Speed Aerodynamics. McGraw Hill, New York.

Kim, B., Shin, Y., 2003. A NURBS panel method for three-dimensional radiation and 
diffraction problems. Journal of Ship Research 47(2), 177-186.

Kim, G.D., Lee, C.S., Kerwin, J.E., 2007. A B-spline based higher-order panel method for the analysis of steady flow around marine propellers, Ocean Engineering 34, 2045-2060.

Kress R. 1989. Linear Integral Equations. Springer Verlag, Berlin.

Kring, D.C., 1995, Numerical stability analysis for time-domain ship motion simulations. Journal of Ship Research 39(4), 313-320.

Lamb H., 1932. Hydrodynamics (6th edition), Cambridge Univ. Press.

Li K., Qian X., 2011. Isogeometric analysis and shape optimization via boundary integral, Computer-Aided Design 43, 1427-1437.

Maniar, H., 1995. A three dimensional higher order panel method based on B-splines. PhD thesis, Massachusetts Inst. of Technology.

Marr, G., Jackson, P.S., 1999. Some improvements and comparisons in the solution of the Neumann-Kelvin problem, J. Ship Res. 43(3), 170-179.

Maskew, B, Hughes, M., Cao Y., 1997. Interim Report USAERO/FSP validation, Anal. Methods Inc, Redmont, Washington.

Mikhlin, S.G., 1965. Multi Dimensional Singular Integrals and Integral Equations, Pergamon Press.

Milne-Thomson, L.M., 1968. Theoretical Hydrodynamics (5th edition), MacMillan.

Mori K. et al, 1994. Proceedings of CFD Workshop - program 2: Inviscid flow around series 60 with free-surface. Tokyo.

Nakos D., Sclavounos P., 1990. On steady and unsteady ship wave patterns. Journal of Fluid Mechanics 215, 263-288.

Nakos D., Sclavounos P., 1994. Kelvin wakes and wave resistance of cruiser and transom stern ships. Journal of Ship Research 38(1), 9-29.

Newman J.N., 1987. Evaluation of the wave-resistance Green function: Part 1-The double integral, J. Ship Res. 31, 2, 79-90. 
Newman J.N., 1987. Evaluation of the wave-resistance Green function: Part 2 - The single integral on the centerplane, J. Ship Res. 31, 79-90.

Okan, M.B., Umpleby, S.M., 1985, Free surface flow around arbitrary two-dimensional bodies by B-splines, International Shipbuilding Progress 32, 182-187.

Paris F. , Canas J., 1997. Boundary Element Methods. Oxford University Press.

Peltzer T.J., Rosenthal B.J., Reppun, W.K., Kring D.C, Milewski W.M., Connell, B., 2008, Multi-body seakeeping design optimization, Proc. 8th Int. Conf. Hydrodyn. (ICHD2008).

Piegl L. and Tiller W. 1997. The NURBS Book. Springer Verlag.

Politis, C, Ginnis, A., Kaklis, P., Belibassakis, K., Feurer, C., 2009. An isogeometric BEM for exterior potential-flow problems in the plane, Proc. SIAM/ACM Joint Conference on Geometric and Physical Modeling, San Francisco, California (art.no.1629302, 349-354).

Press, W. H., Flannery, B. P., Teukolsky, S. A., and Vetterling, W. T., 1992. Numerical Recipes in FORTRAN: The Art of Scientific Computing, Cambridge University Press.

Raven H.C., 1996. A solution method for the nonlinear ship wave resistance problem PhD-thesis, Delft University of Technology.

Shampine L.F. 2008. Vectorized Adaptive Quadrature in MATLAB, J of Computational and Applied Mathematics 211, 131-140.

Telles, J.F.C., 1987. A self-adaptive co-ordinate transformation for efficient numerical evaluation of general boundary element integrals, Int. Journal Numerical Meth. in Engineering 24, 959-973.

Telles, J.F.C., and Oliveira, R.F., 1994. Third degree polynomial transformation for boundary element integrals: Further improvements, Engin. Analysis Boundary Elements $13,135-141$.

Voutsinas S., Bergeles, G., 1990. Numerical calculation of singular integrals appearing in 3D potential flow problems, Appl.Math.Modelling 14, 618-629.

Wehausen J.V., 1973. The wave resistance of ships, Advances in Applied Mechanics 13, 93-245. 


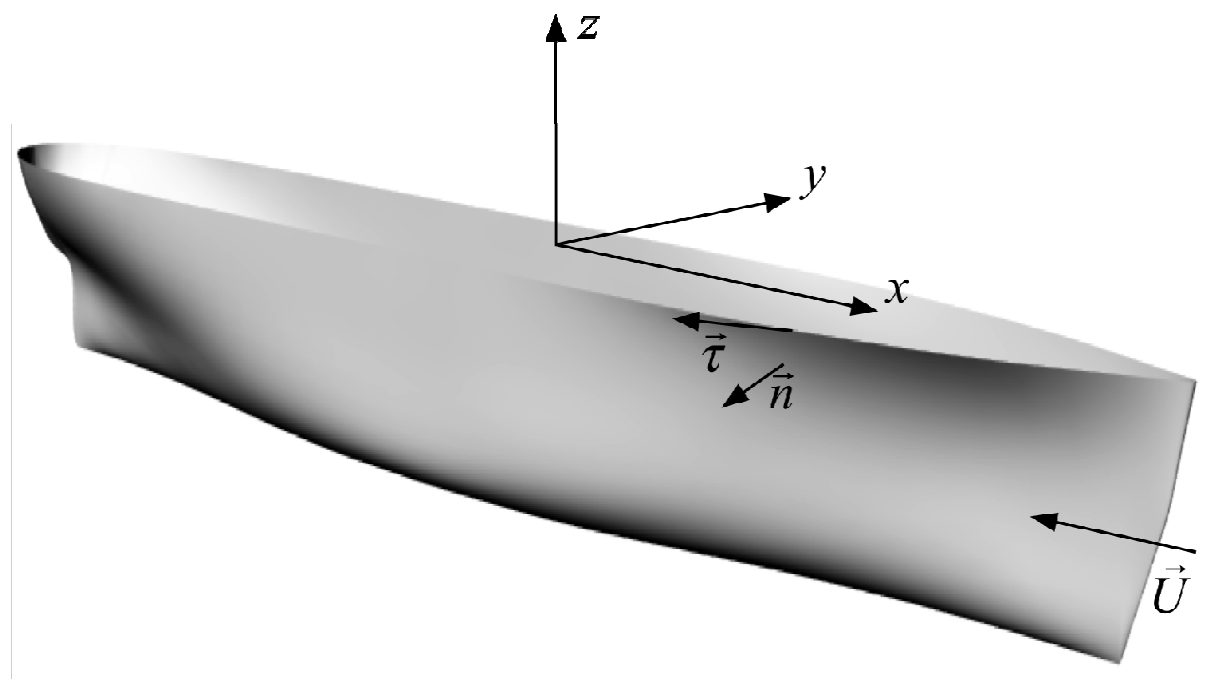

Figure 1. Ship in a uniform stream with velocity $\mathbf{U}=(-U, 0,0)$. 

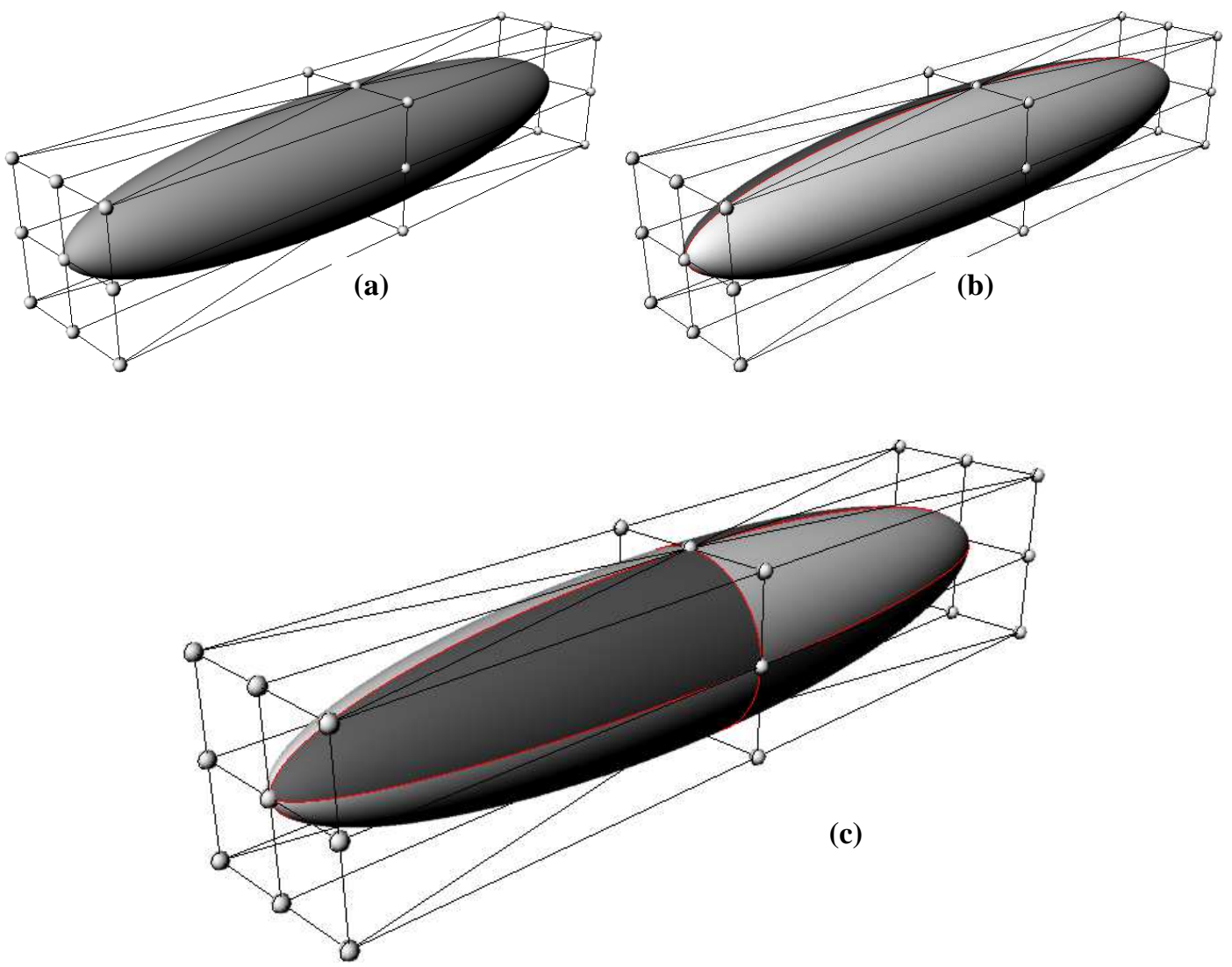

Figure 2. Multi-patch NURBS representation of a 5:1:1 prolate spheroid using a) one , b) two and c) four patches. The control net is depicted by connecting with linear segments the control points (gray spheres). 

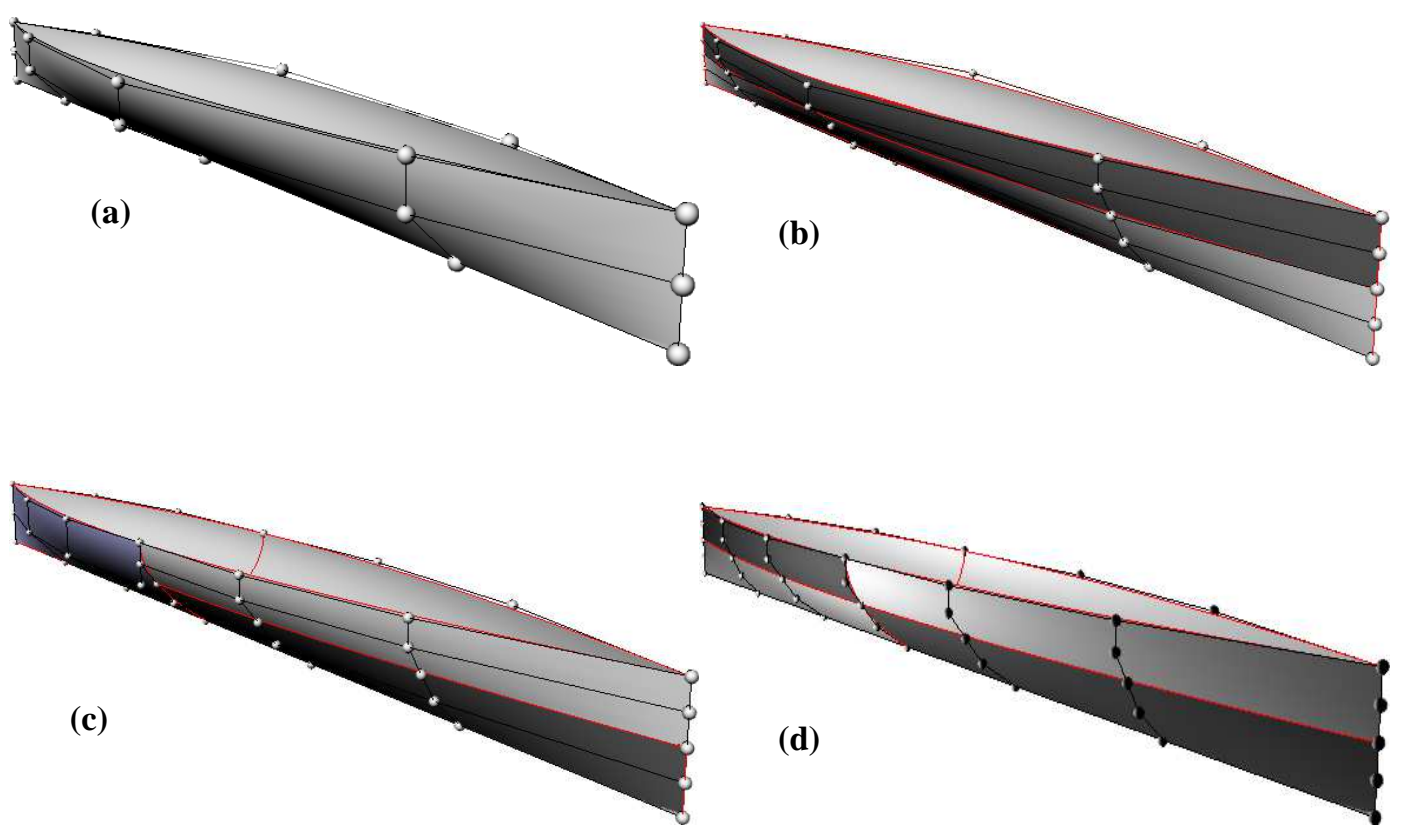

Figure 3. Multi-patch B-spline representation of a Wigley hull using a) one, b) two , c) three and d) four patches. 
(a)

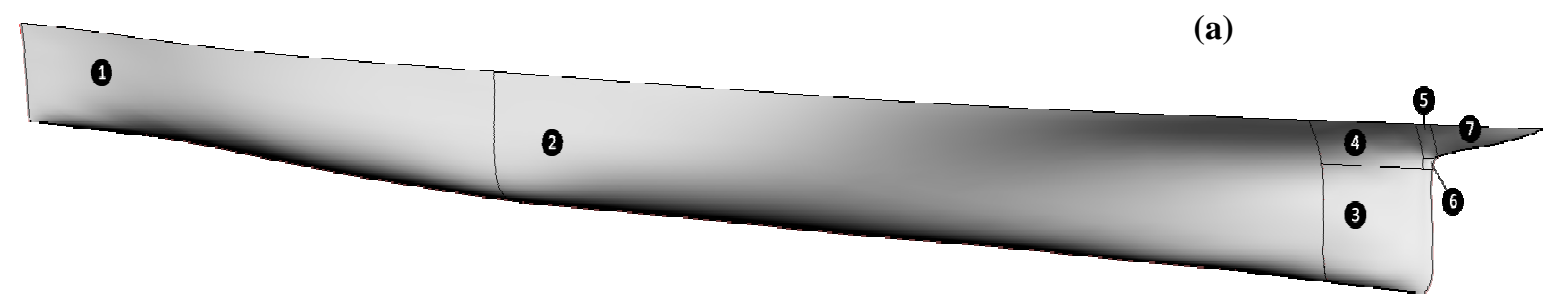

(b)

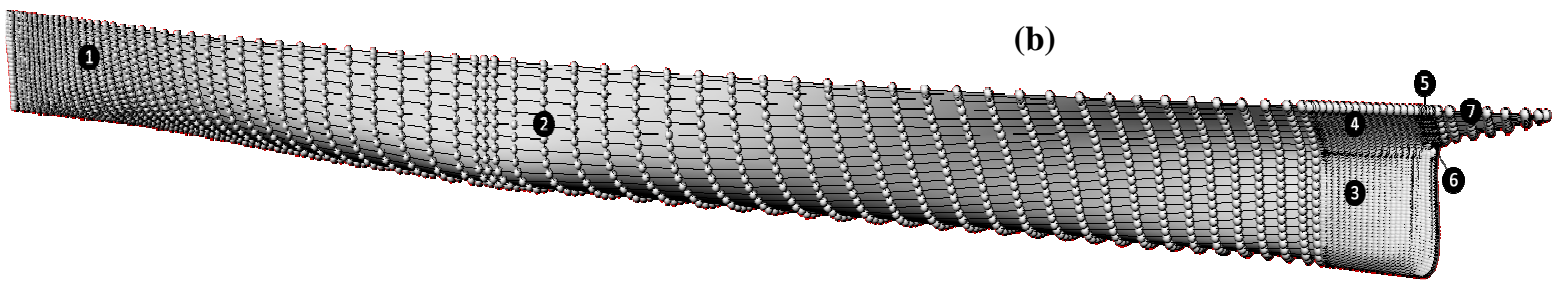

(c)

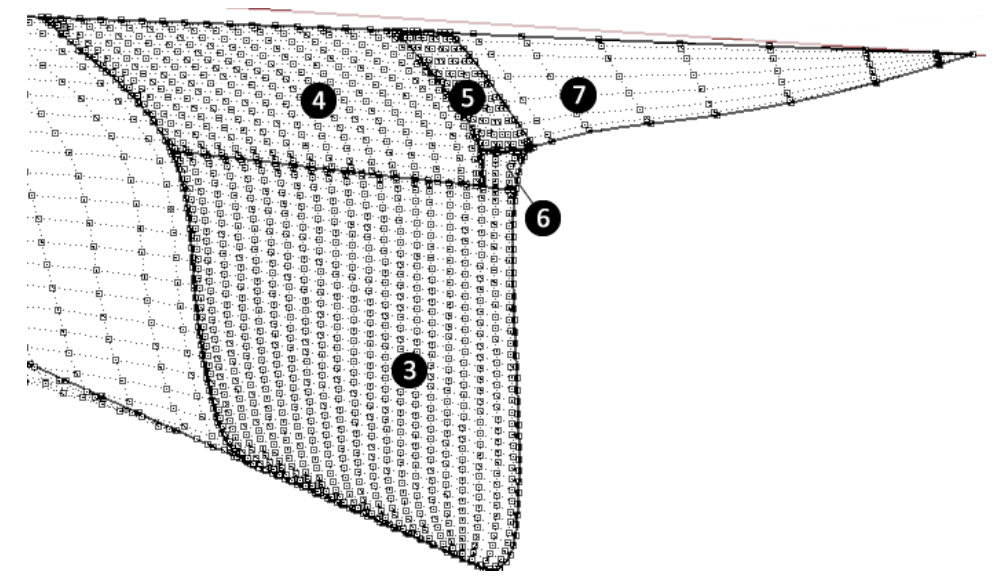

Figure 4. (a) A multi-patch (using 7 patches) $G^{1}$-representation of a Series $60, \mathrm{Cb}=0.60$ hull, and (b) its control net. A zoom on the stern part (c) is also included. 
(a)

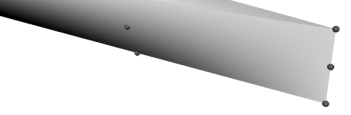

(b)

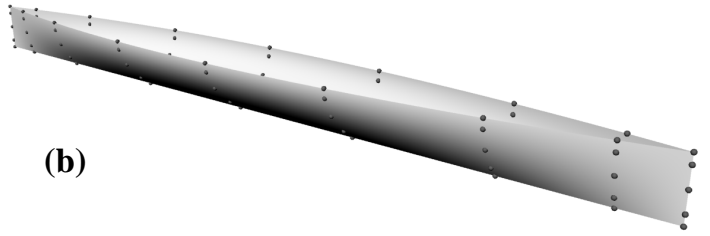

(c)

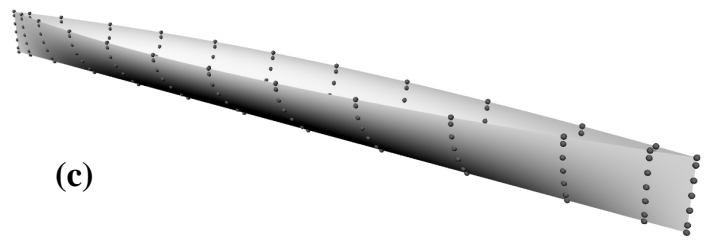

Figure 5. Greville collocation-points distribution on the Wigley hull using a) the original knot vectors and those resulting after insertion of b) 2 and c) 4 knots per parametric interval. 

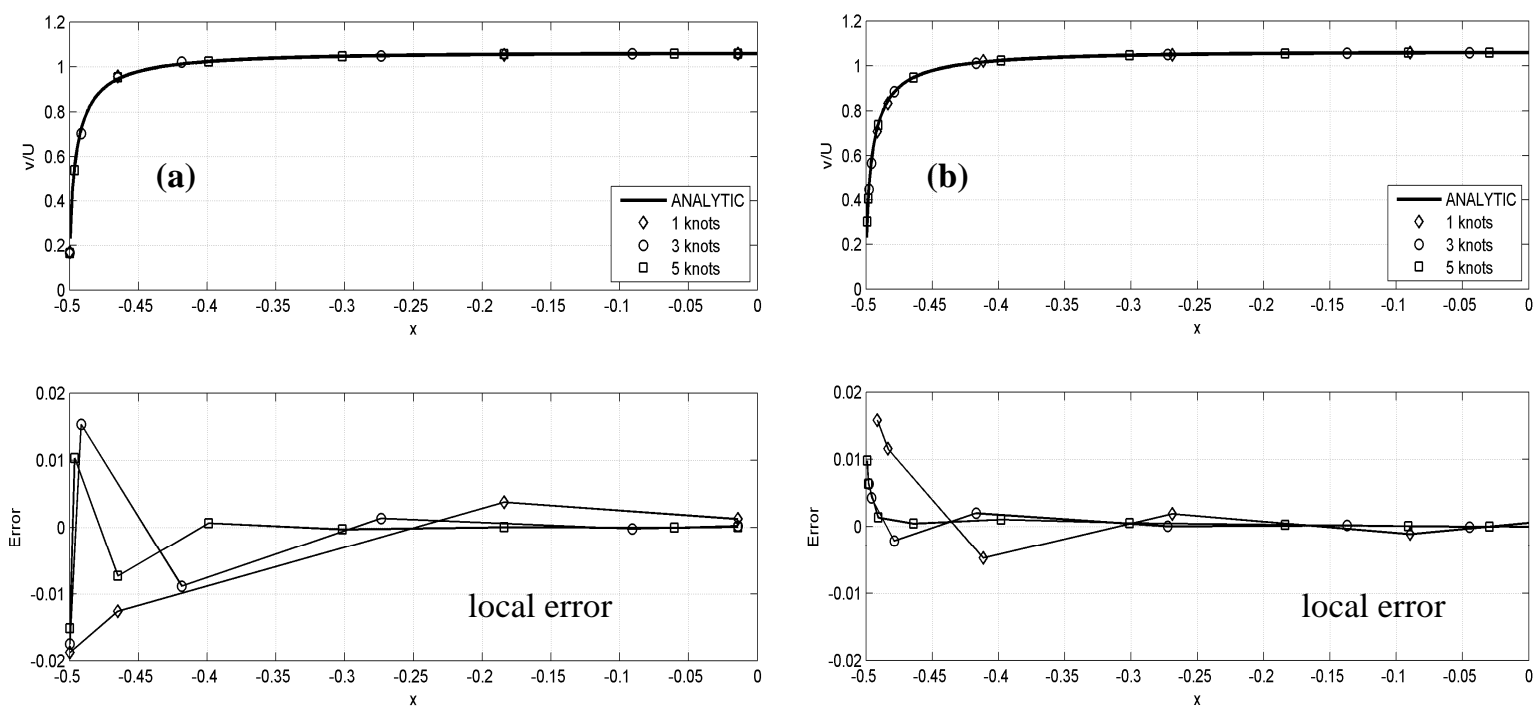

Figure 6. Velocity distribution along the top meridian of the $x z$-plane of a prolate spheroid (axes-ratio: 5:1:1) in infinite domain, for parallel inflow along its $x$-axis. Comparison of the analytical solution versus the results obtained by using (a) $x$-axis and (b) $z$-axis parameterizations. The corresponding local error along the same meridian is shown in the lower subplots. 

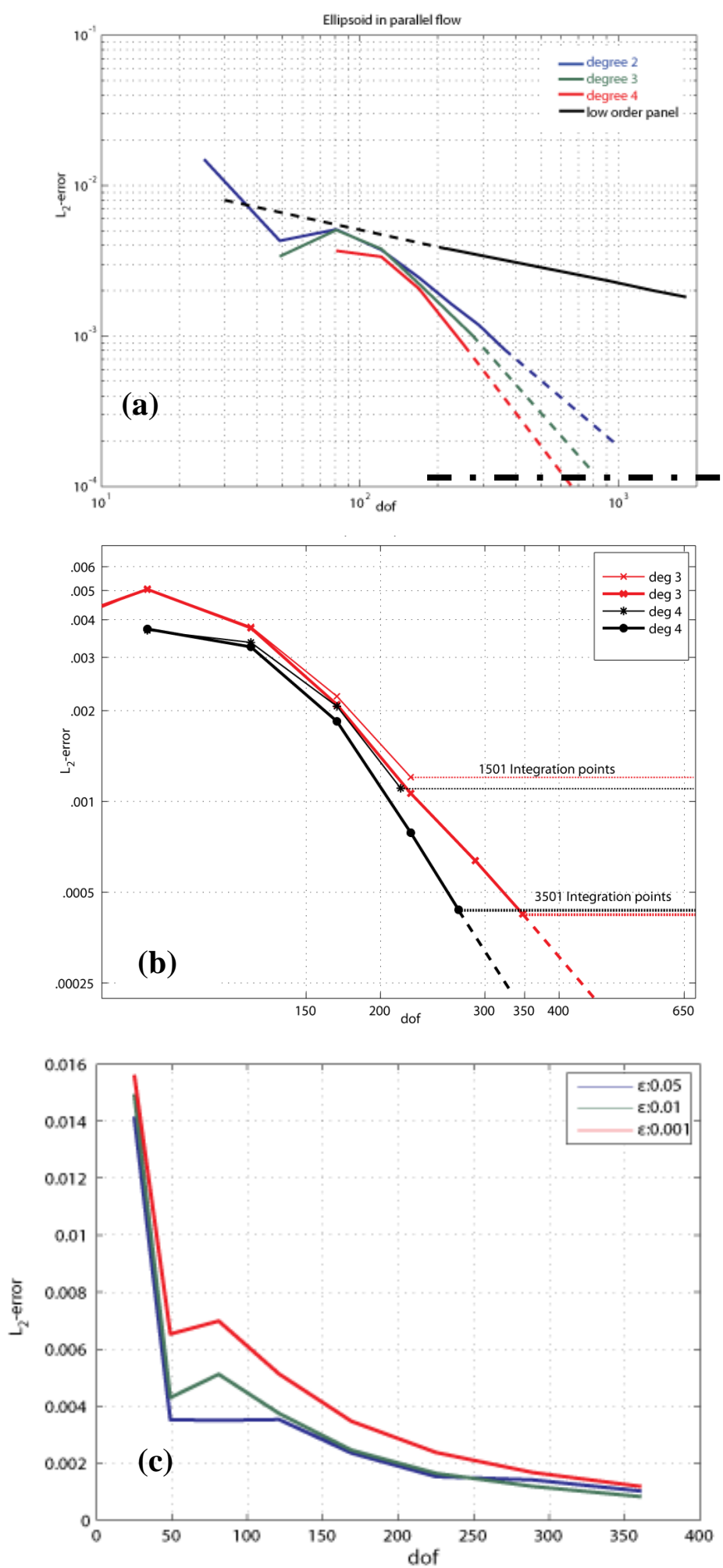

Figure 7. Ellispsoid in parallel flow. (a) Decay of the $\mathrm{L}^{2}$-error with respect to dof and degree elevation. The corresponding prediction, obtained by the low-order panel method, is also shown using a black line. Plateau level is indicated by the thick dashed-dot line. (b) Dependence of the plateau level on the numerical-integration accuracy (1501 and 3501 integration points are used). (c) Sensitivity analysis of the error due to slight shifting $(\varepsilon=0.05,0.01,0.001)$ of the collocation points, in the case of quadratic NURBS 

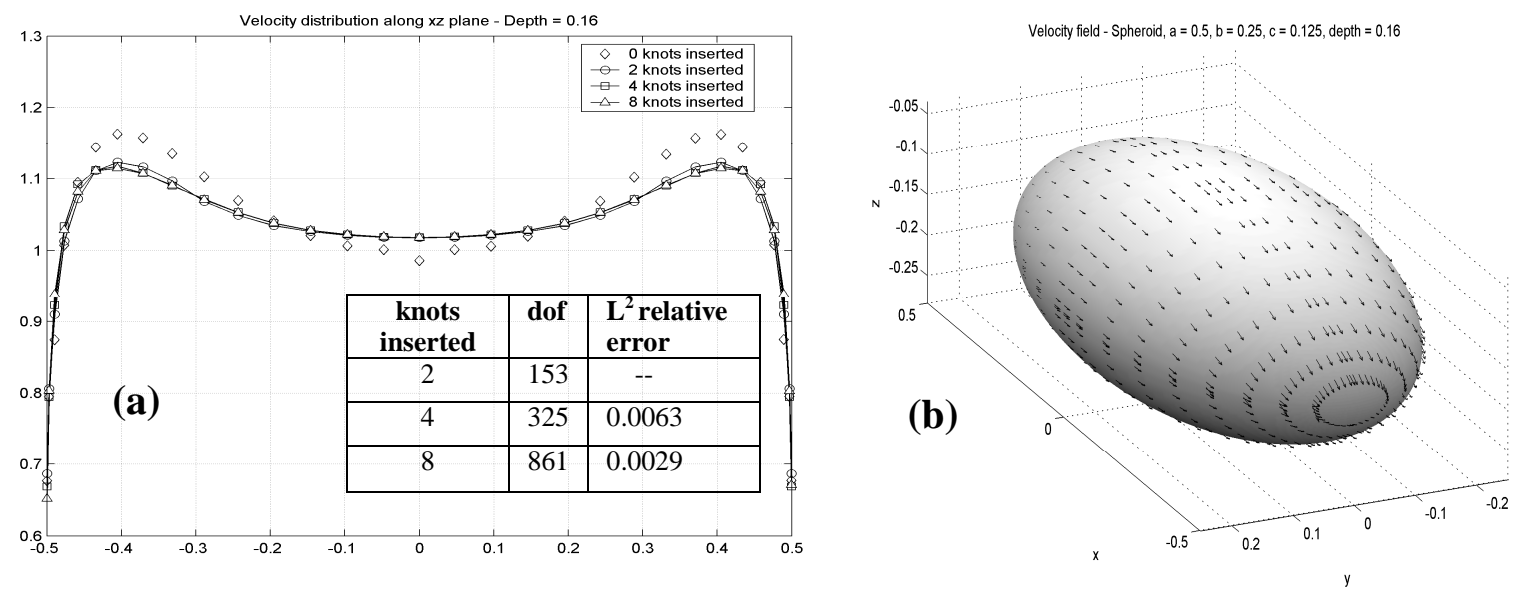

Figure 8. Velocity field on the surface of 3-axial ellipsoid (axes ratio: 2:1:0.5), at low submergence $d / L=0.16$, using homogeneous Dirichlet boundary condition on the undisturbed free surface. Horizontal velocity along the top meridian (left) and 3D vector plot of surface velocities (right). 


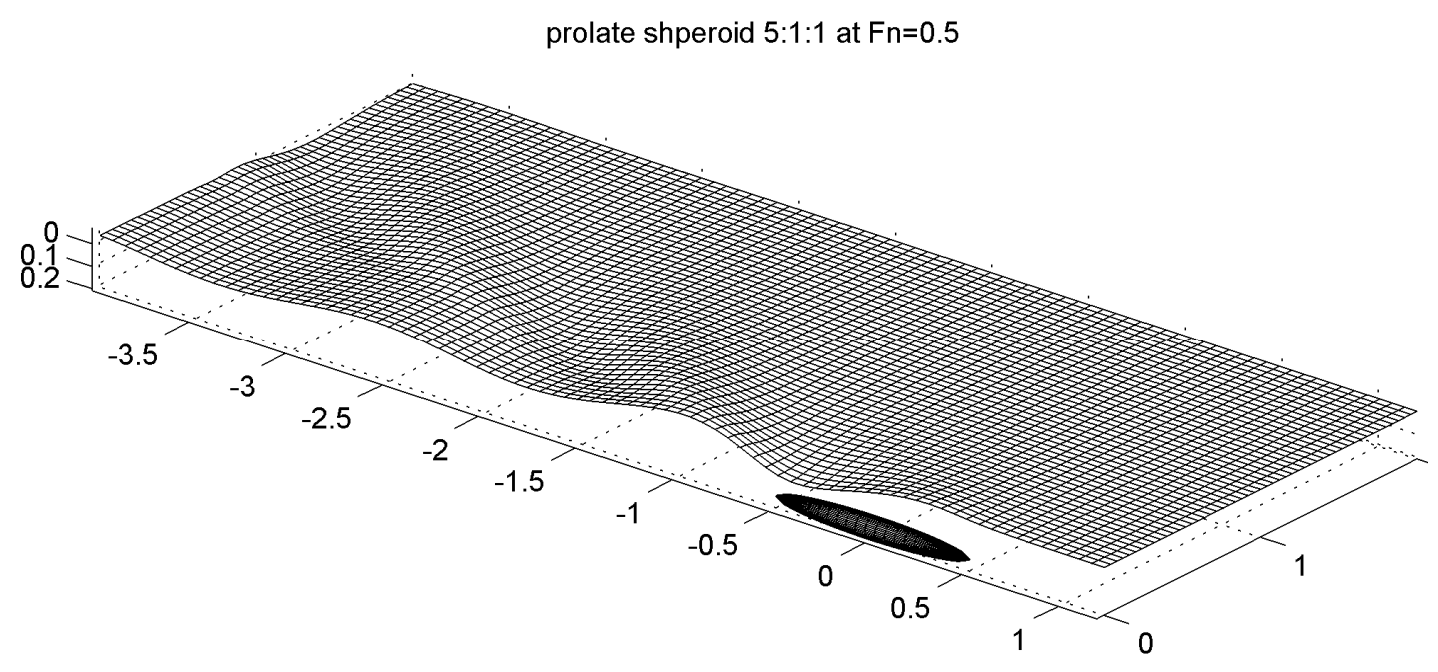

Figure 9. Free-surface elevation generated by a prolate spheroid with axes ratio 5:1:1, translating steadily at low submergence $(d / L=0.16)$ with Froude number $F=0.5$. 


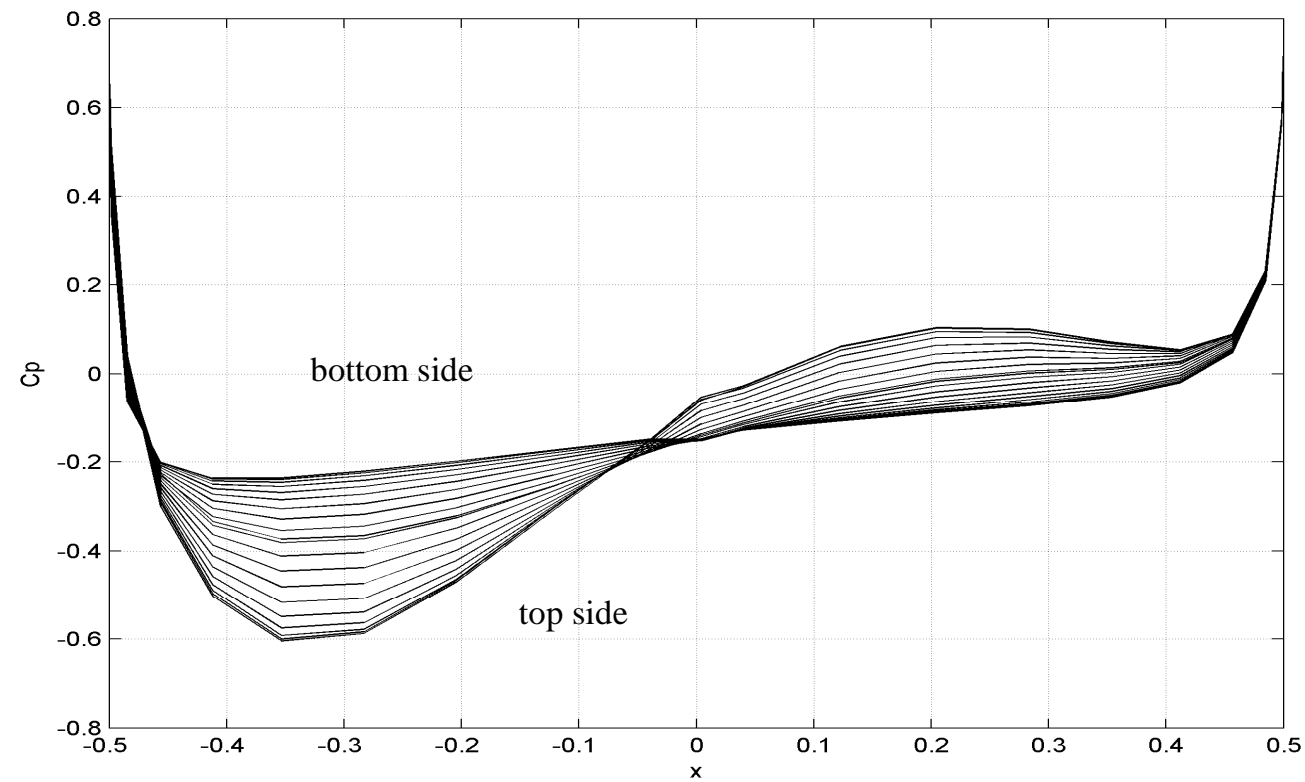

Figure 10. Pressure coefficient $C_{p}$ along a series of meridians of the prolate spheroid in Fig. 9. 


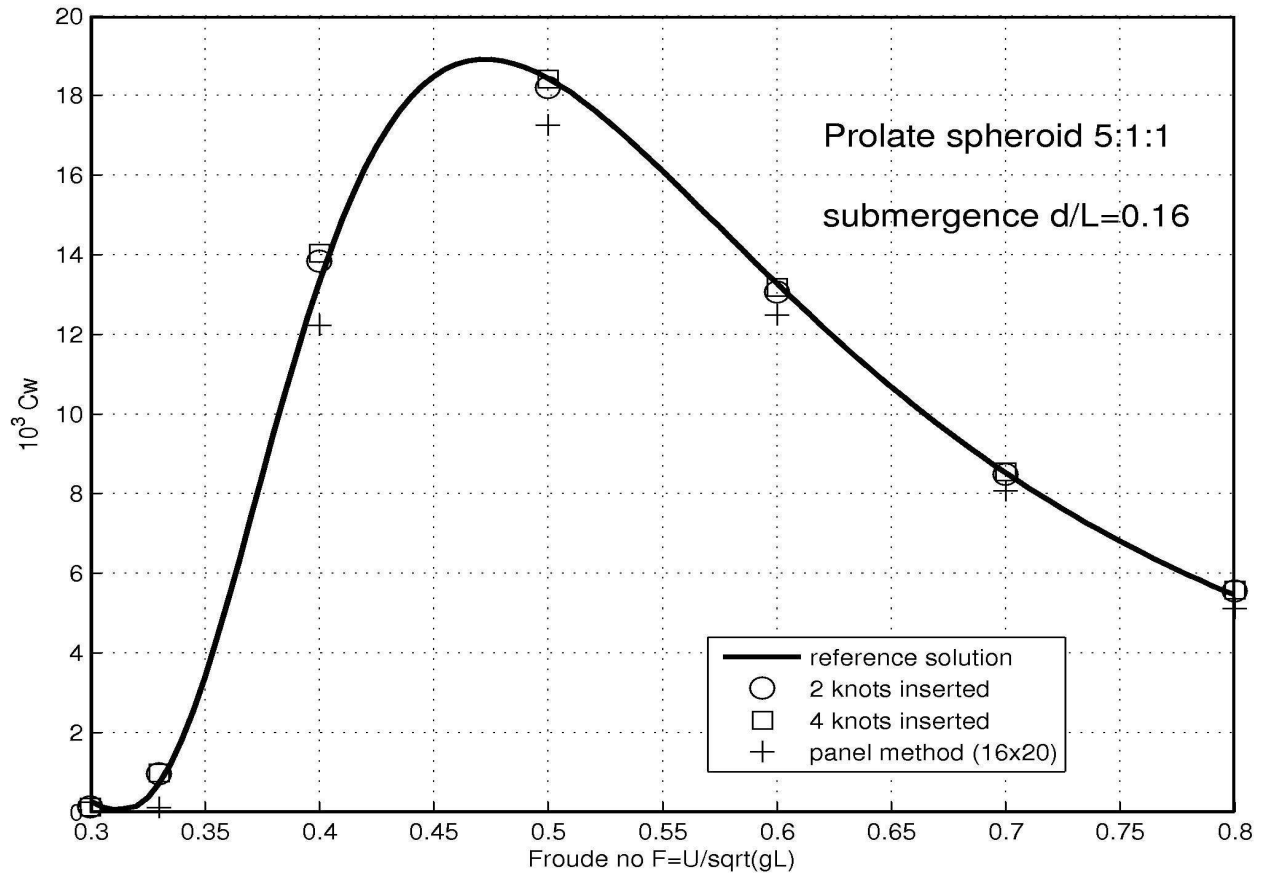

Figure 11. Wave-resistance coefficient $C_{W}$ of the prolate spheroid in Fig. 9, for various Froude numbers. 

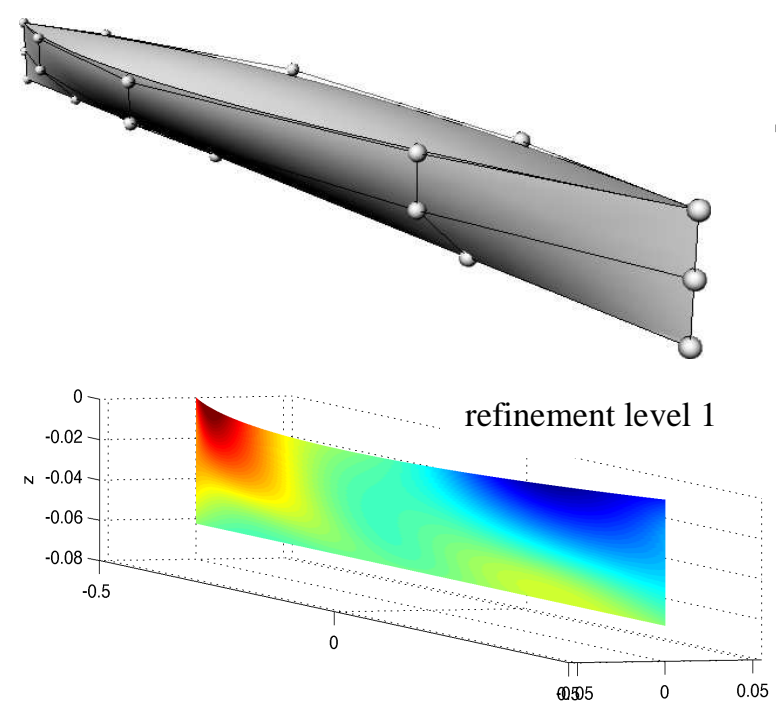
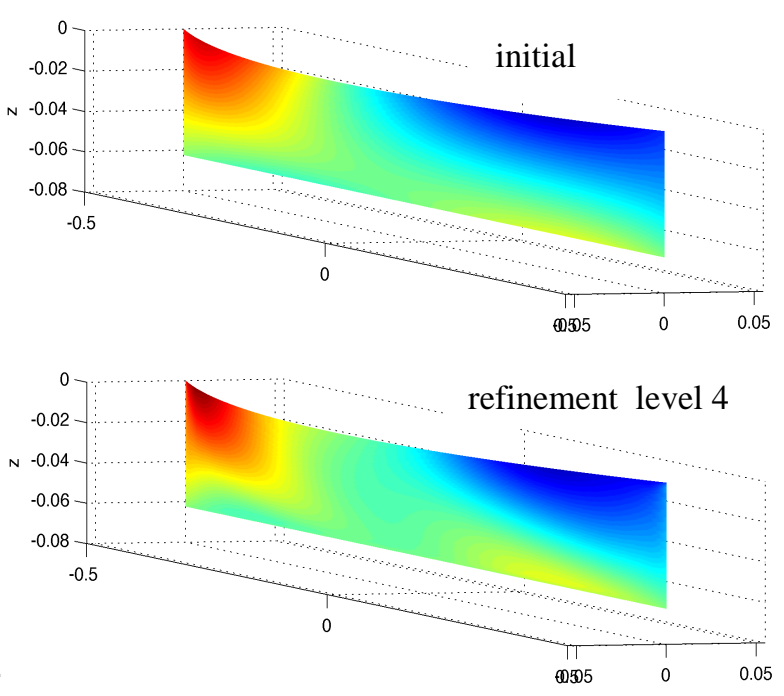

0.3

Figure 12. Source-sink distribution obtained at refinement levels 0 (initial), 1 and 4 for the singlepatch representation of the Wigley hull, at $F=0.316$. The upper-left part of the figure depicts the control net of the single-patch representation of the hull. 

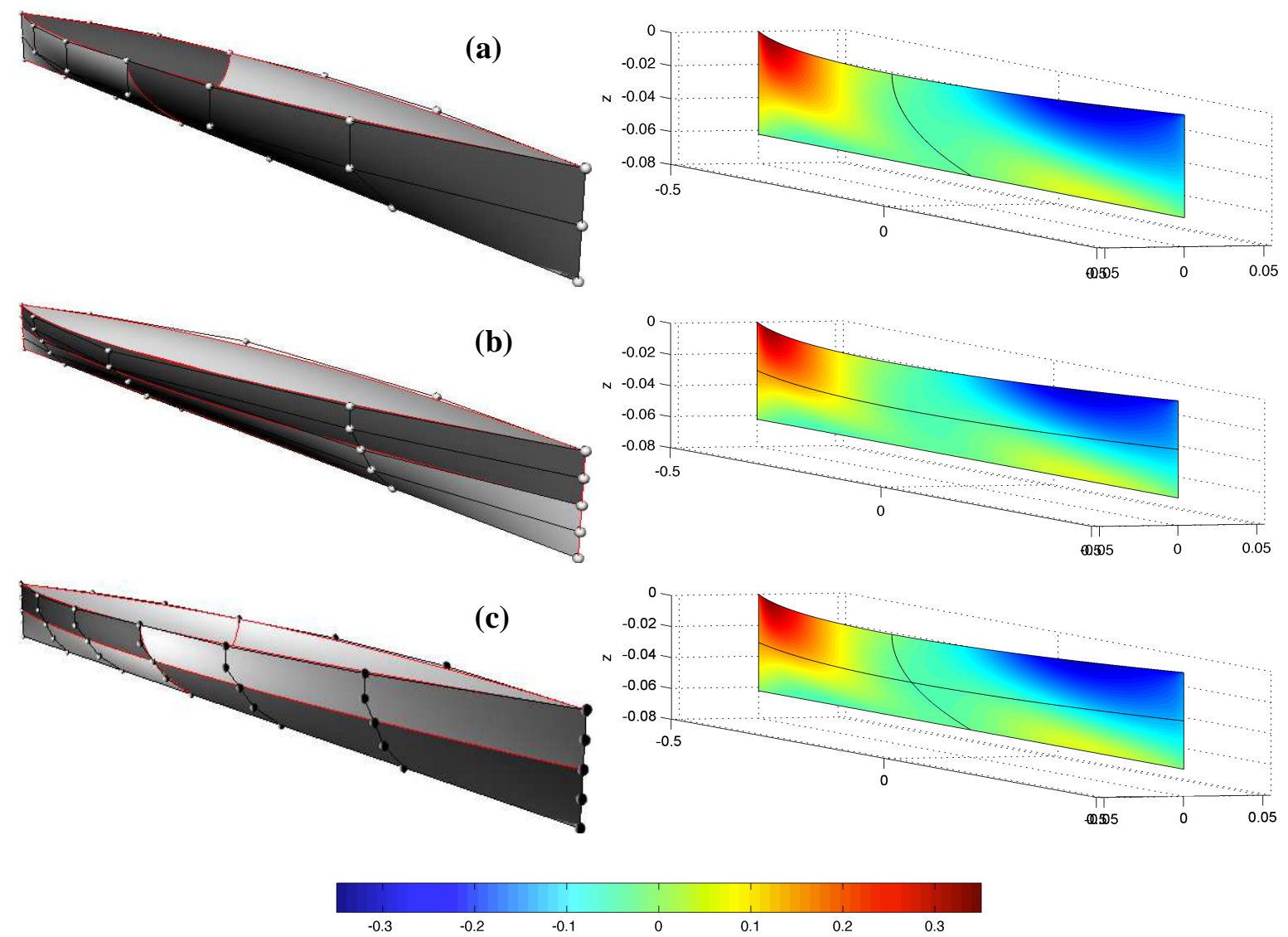

Figure 13. Source-sink distribution obtained at the same refinement level (4), for three multi-patch representations of the Wigley hull $(F=0.316)$ : (a) two vertical patches, (b) two horizontal patches, (c) four patches. The left part of the figure depicts the control nets of the three alternative multi-patch representations of the hull. 


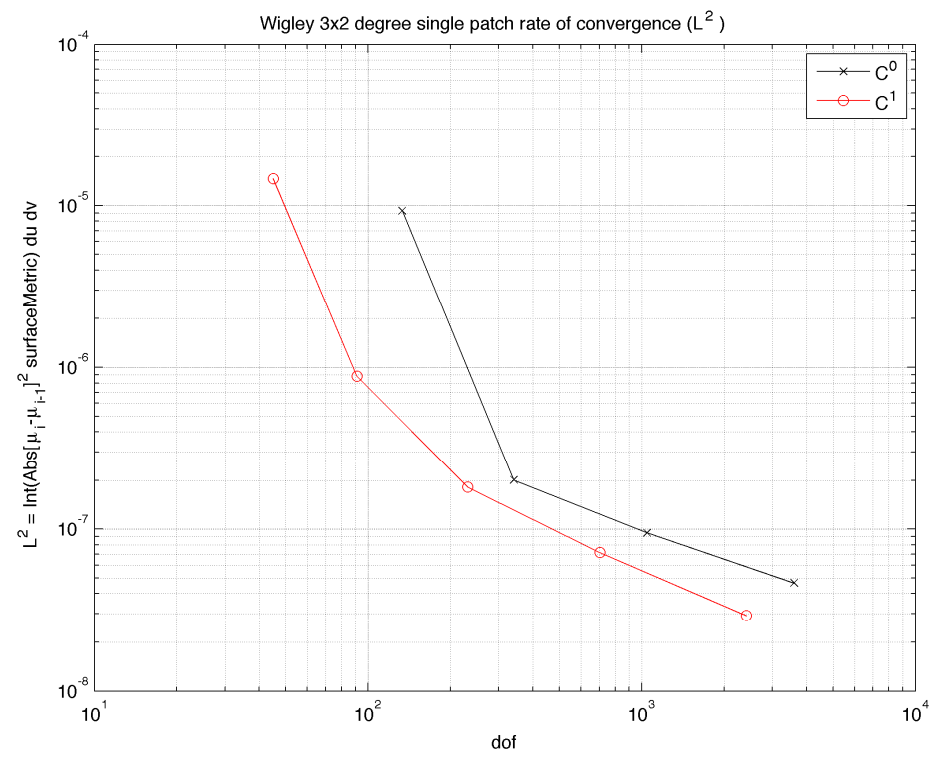

Figure 14. Relative $\mathrm{L}^{2}$ error $\left(\left\|\mu_{i+1}-\mu_{i}\right\|\right.$, where $\left.i=d o f\right)$ of the numerical solution as obtained by the present IGA-BEM (shown by using red lines) against corresponding results obtained by bi-quadratic Lagrange BEM (black lines), in the case of the Wigley hull. 


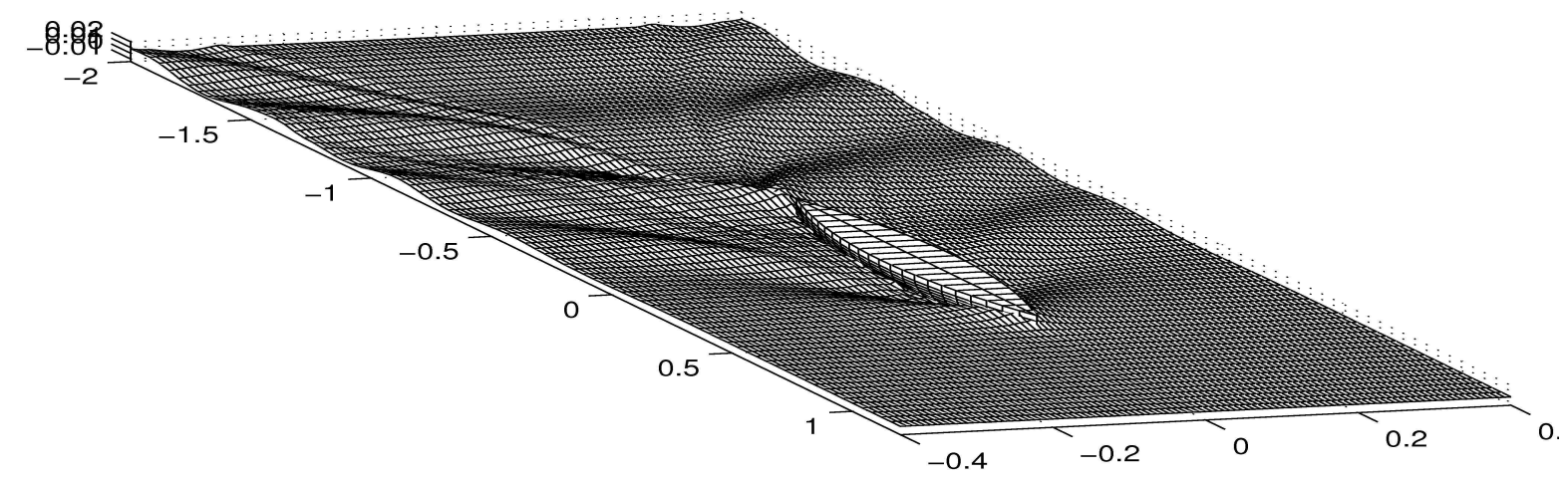

Figure 15. Free-surface elevation generated by the Wigley hull, at Froude number $F=0.316$, as calculated by the present method using a single-patch representation 

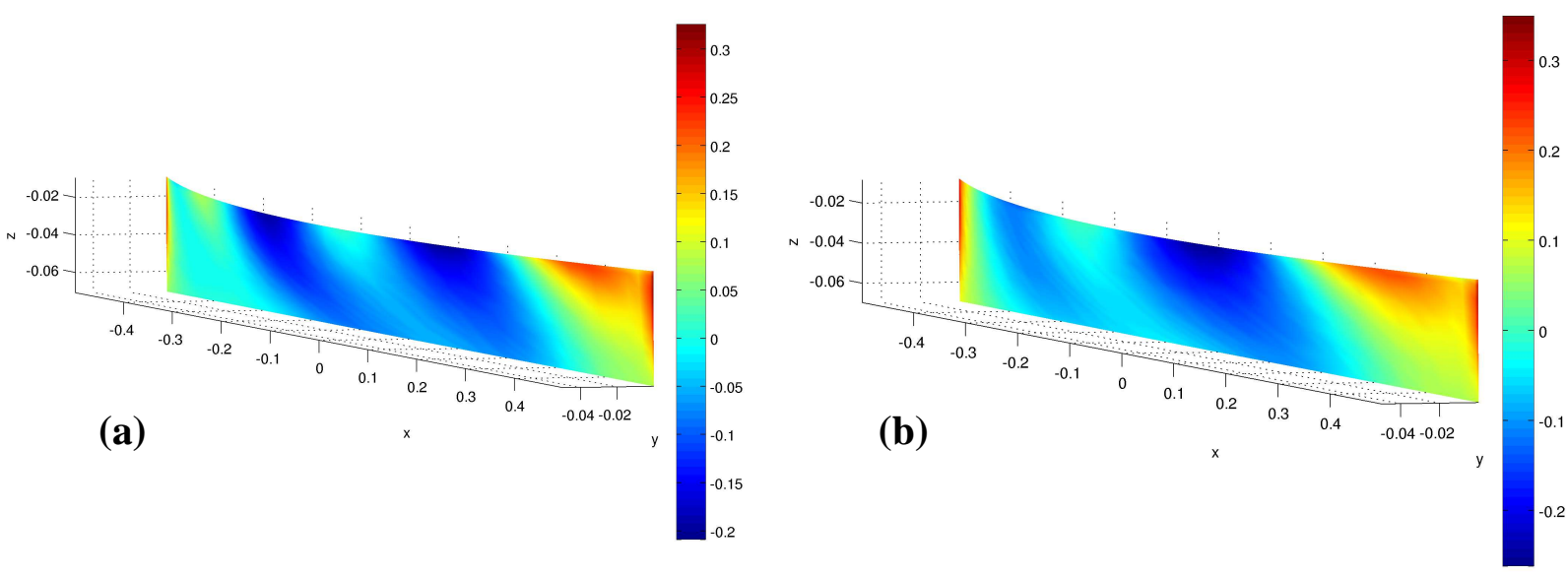

Figure 16. Pressure coefficient $\mathrm{Cp}$ on the Wigley hull, for (a) $F=0.267$ and (b) $F=0.316$, as calculated by the present method using dof $=703$ (single patch, refinement level 4). 


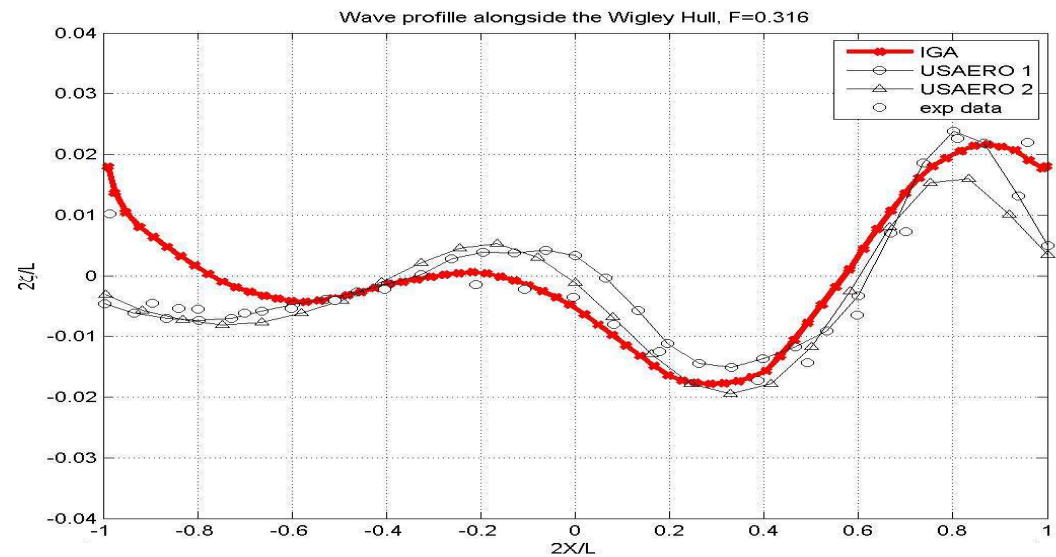

Figure 17. Wave profile at $F=0.316$ alongside the Wigley hull. Comparison with experimental data and computations by other panel methods. 


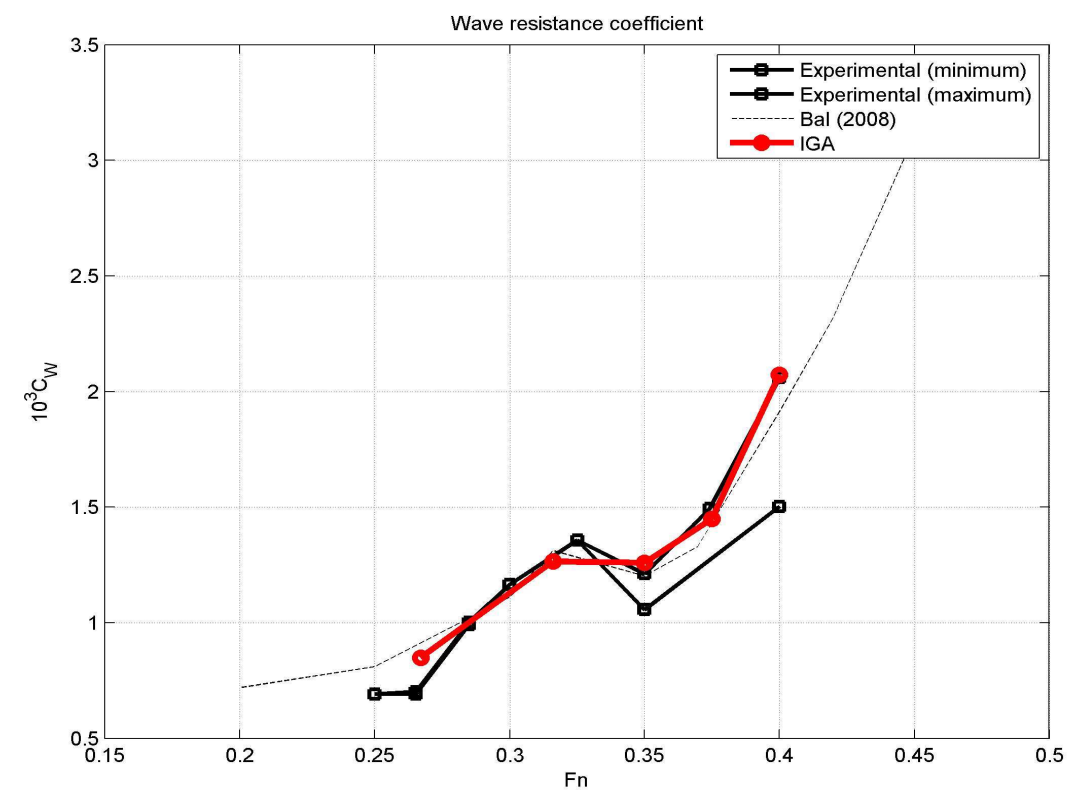

Figure 18. Wave resistance coefficient $C_{W}$ of the Wigley hull for various Froude numbers, as calculated by the present method (red bullets). Comparison with experimental data (black squares) and another panel method (thin dashed curve). 


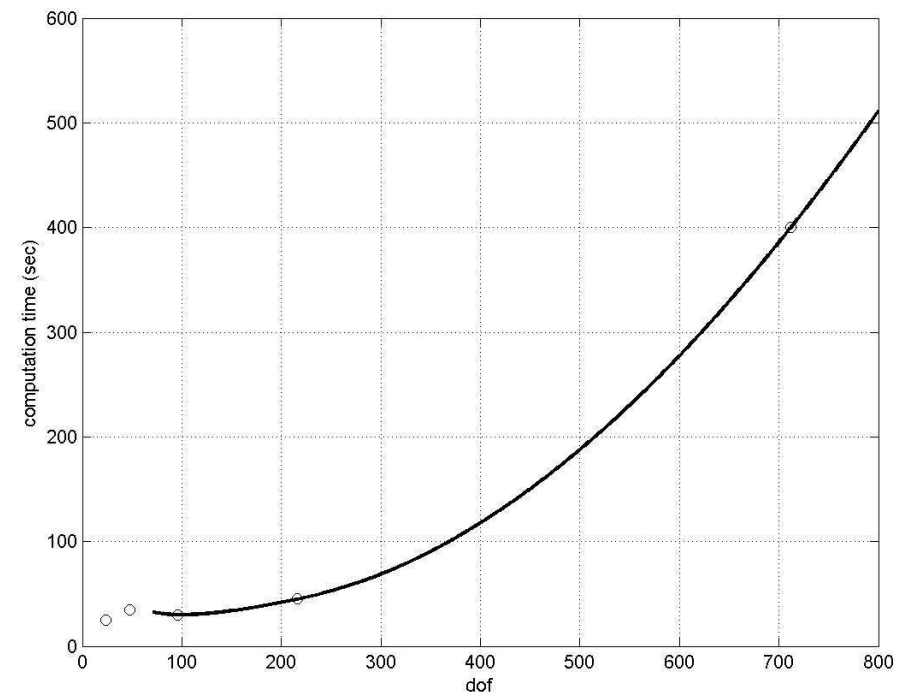

Figure 19. Computation time versus dof in the case of the Wigley hull using the present IGA-BEM. 


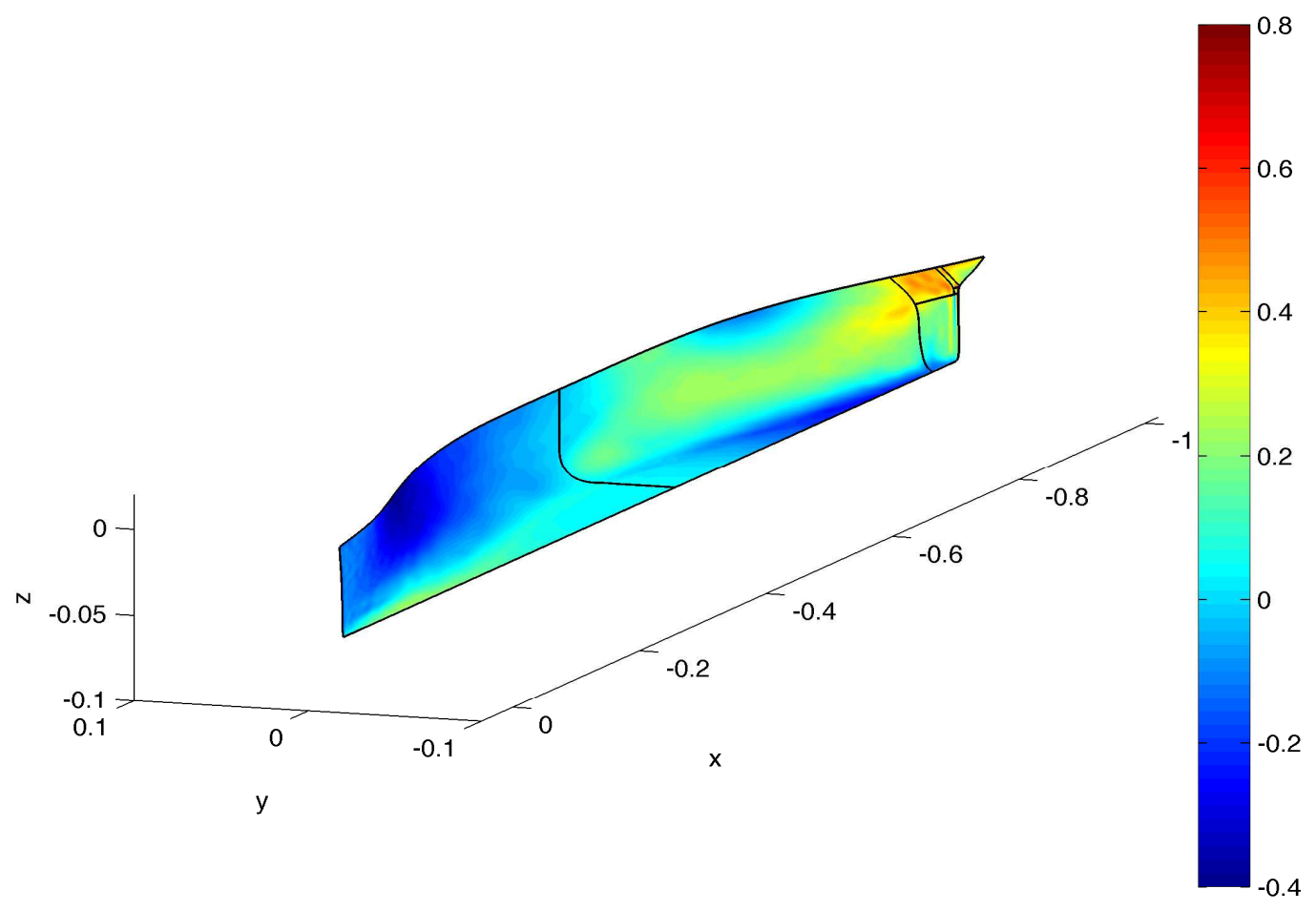

Figure 20. Source-sink distribution on the surface of the Series $60\left(C_{b}=0.60\right)$ hull at Froude number $F=0.316$. 


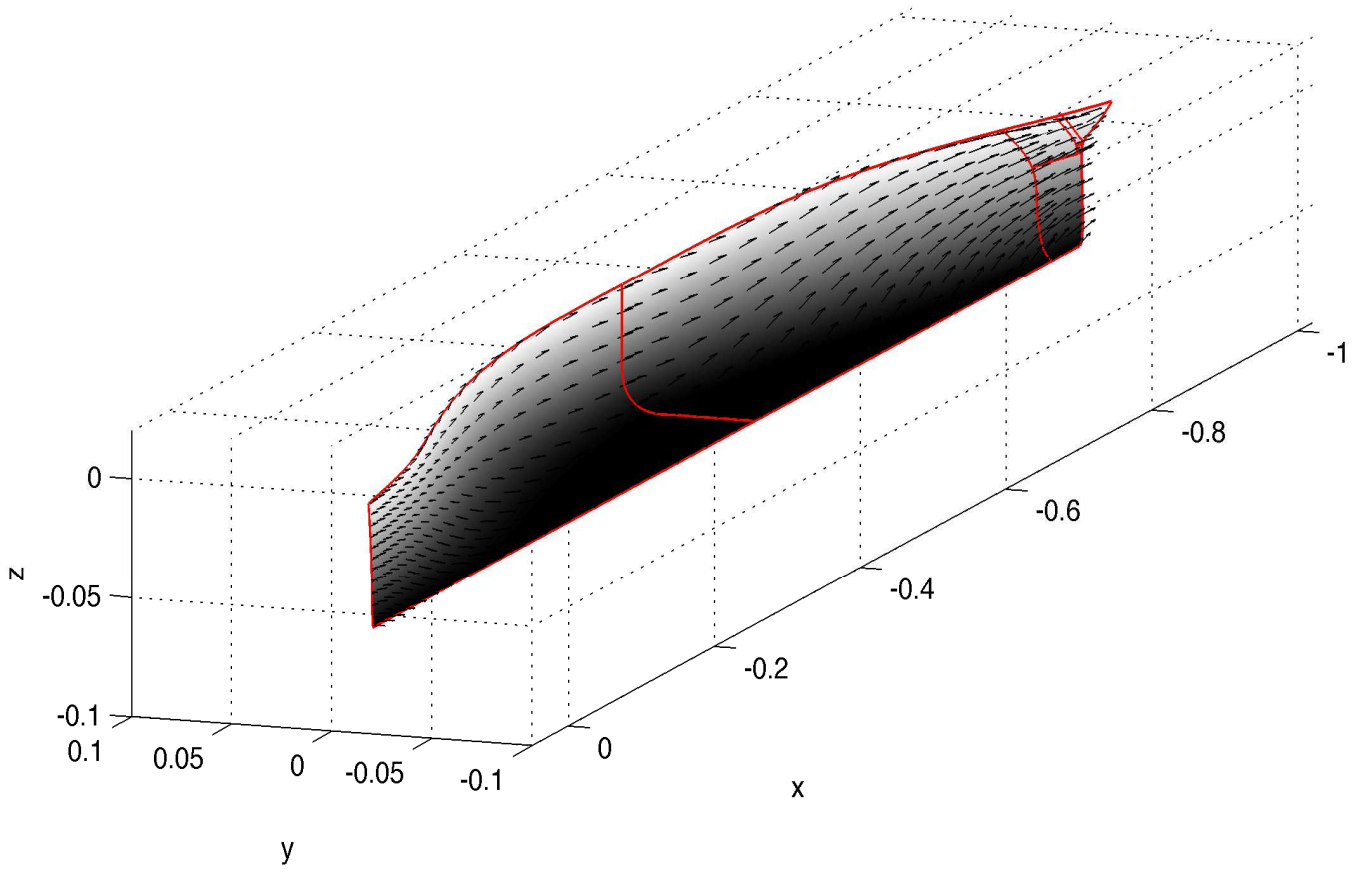

Figure 21. Surface velocity distribution on the surface of the Series $60 \quad\left(C_{b}=0.60\right)$ hull at Froude number $F=0.316$. 


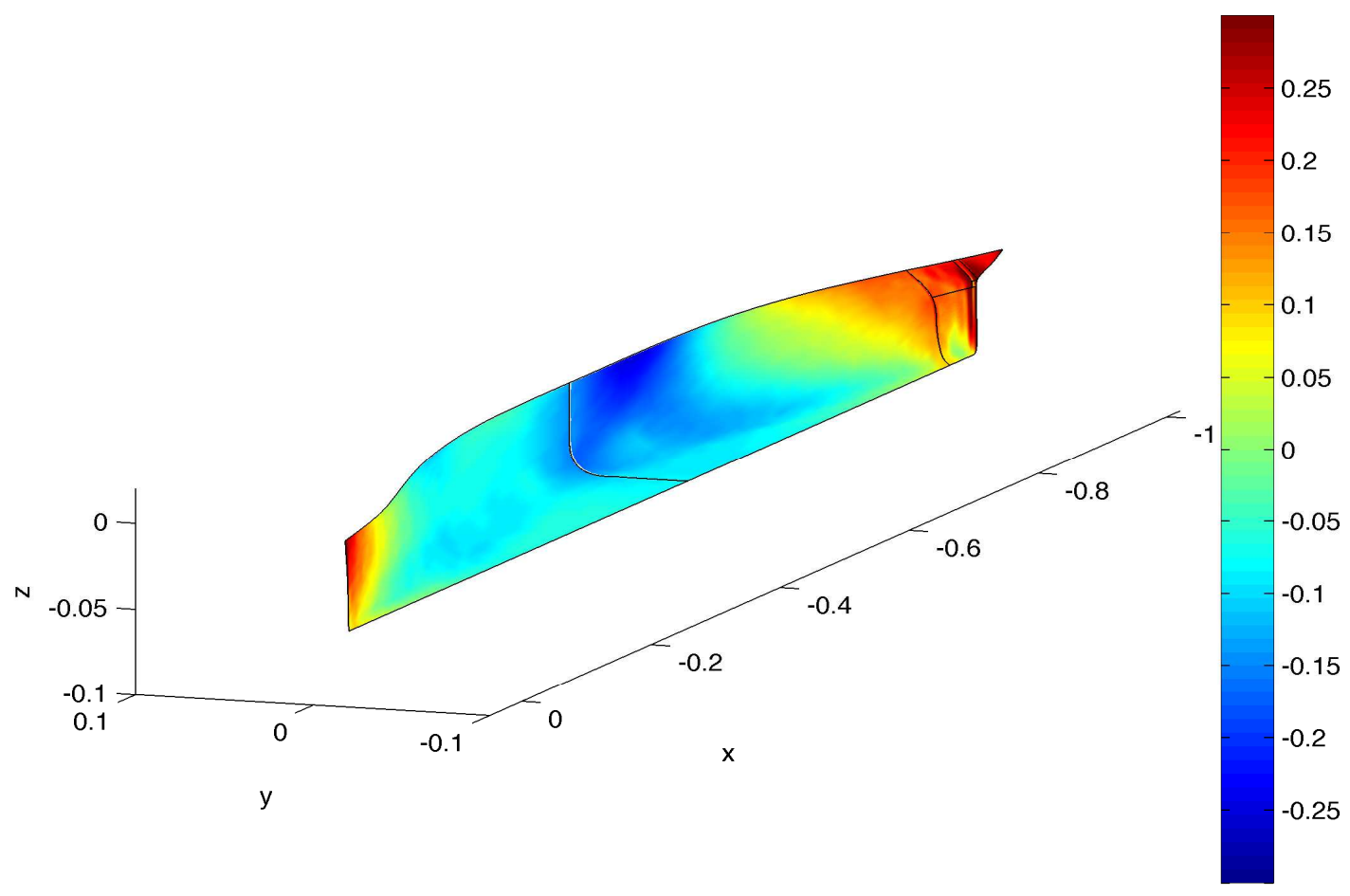

Figure 22. Distribution of the pressure coefficient $C_{P}$ on the surface of the Series 60 $\left(\mathrm{C}_{\mathrm{b}}=0.60\right)$ hull at Froude number $F=0.316$. 


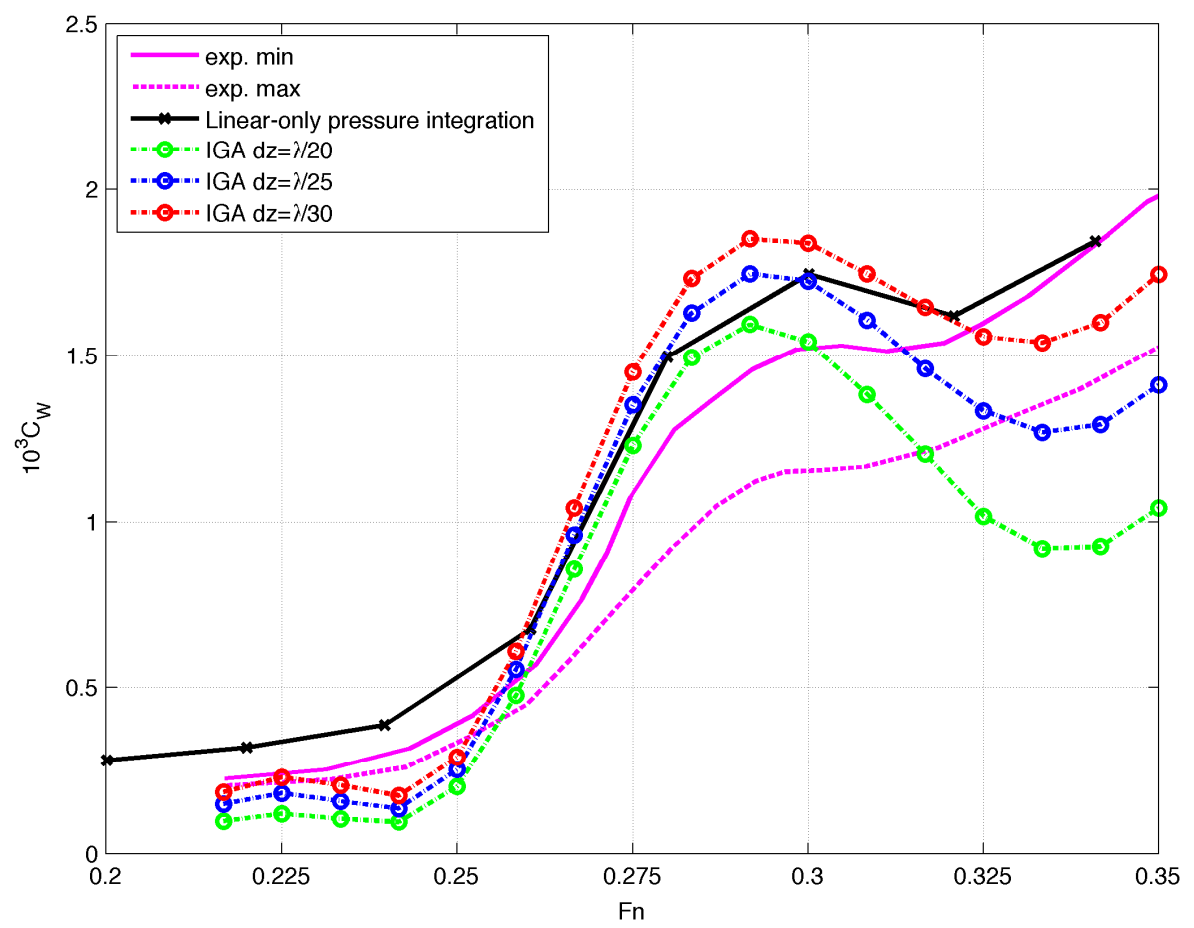

Figure 23. Wave resistance coefficient $C_{W}$ of the Series-60 $\left(\mathrm{C}_{\mathrm{b}}=0.60\right)$ hull for various Froude numbers, as calculated by the present method (using different values for the submergence $\delta z$ ). Comparison with experimental data (shown by cyan lines) and the BEM by Nakos \& Sclavounos (1994), shown by solid black line. 


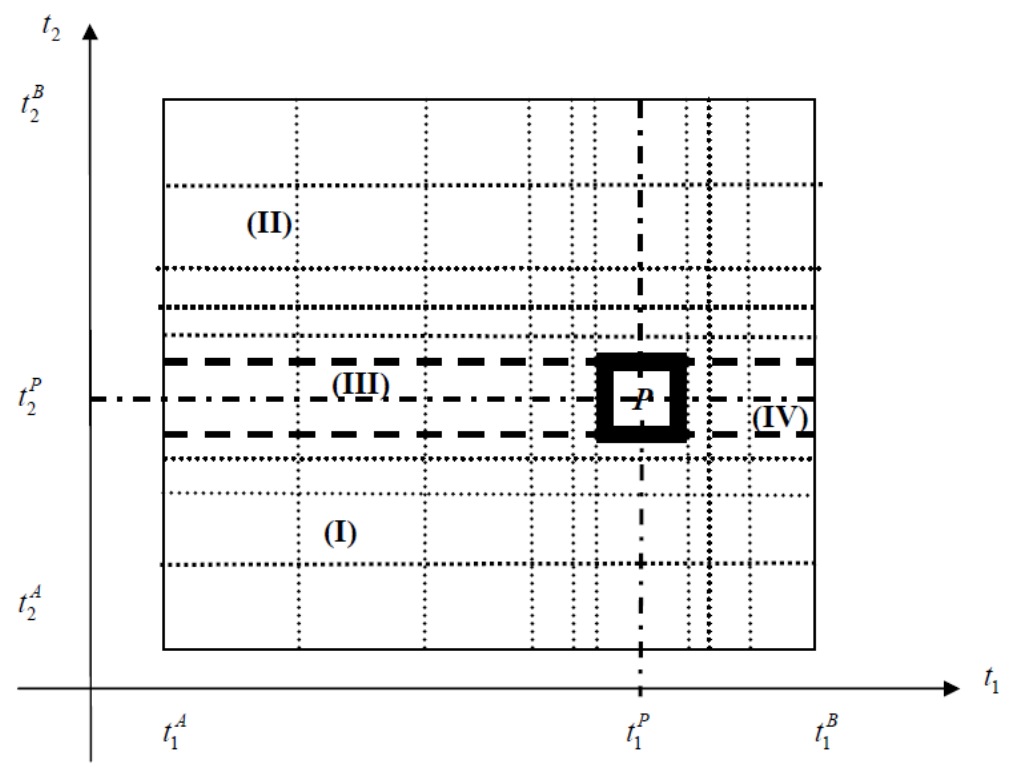

Figure A1: Numerical grid for the calculation of the 2D Cauchy principal value integrals. The $\varepsilon$ - neighborhood, cutting-off the singularity $\left(t_{1}^{P}, t_{2}^{P}\right)$, is denoted by a thick box. 